\title{
Sphere Packings, I
}

\author{
T. C. Hales \\ Department of Mathematics, University of Michigan, \\ Ann Arbor, MI 48109, USA \\ hales@umich.edu
}

\begin{abstract}
We describe a program to prove the Kepler conjecture on sphere packings. We then carry out the first step of this program. Each packing determines a decomposition of space into Delaunay simplices, which are grouped together into finite configurations called Delaunay stars. A score, which is related to the density of packings, is assigned to each Delaunay star. We conjecture that the score of every Delaunay star is at most the score of the stars in the face-centered cubic and hexagonal close packings. This conjecture implies the Kepler conjecture. To complete the first step of the program, we show that every Delaunay star that satisfies a certain regularity condition satisfies the conjecture.
\end{abstract}

\section{Introduction}

The Kepler conjecture asserts that no packing of spheres in three dimensions has density exceeding that of the face-centered cubic lattice packing. This density is $\pi / \sqrt{18} \approx$ 0.74048 . In an earlier paper [H2] we showed how to reduce the Kepler conjecture to a finite calculation. That paper also gave numerical evidence in support of the method and conjecture. This finite calculation is a series of optimization problems involving up to 53 spheres in an explicit compact region of Euclidean space. Computers have little difficulty in treating problems of this size numerically, but a naive attempt to make a thorough study of the possible arrangements of these spheres would quickly exhaust the world's computer resources.

The first purpose of this paper is to describe a program designed to give a rigorous proof of the Kepler conjecture. A sketch of a related program appears in [H2]. Although the approach of [H2] is based on substantial numerical evidence, some of the constructions of that paper are needlessly complicated. This paper streamlines some of those constructions and replaces others with constructions that are more amenable to rigorous methods. For this program to succeed, the original optimization problem must be partitioned into a series of much smaller problems that may be treated by current computer technology or hand calculation. 
The second purpose of this paper is to carry out the first step of the proposed program. A statement of the result is contained in Theorem 1 below.

Background to another approach to this problem is found in [H3]. To add more detail to the proposed program, we recall some constructions from earlier papers [H1], [H2]. Begin with a packing of nonoverlapping spheres of radius 1 in Euclidean three-space. The density of a packing is defined in [H1]. It is defined as a limit of the ratio of the volume of the unit balls in a large region of space to the volume of the large region. The density of the packing may be improved by adding spheres until there is no further room to do so. The resulting packing is said to be saturated. It has the property that no point in space has distance greater than 2 from the center of some sphere.

Every saturated packing gives rise to a decomposition of space into simplices called the Delaunay decomposition. The vertices of each Delaunay simplex are centers of spheres of the packing. None of the centers of the spheres of the packing lie in the interior of the circumscribing sphere of any Delaunay simplex. In fact, this property is enough to determine the Delaunay decomposition completely except for certain degenerate packings. A degeneracy occurs, for instance, when two Delaunay simplices have the same circumscribing sphere. In practice, these degeneracies are important, because they occur in the face-centered cubic and hexagonal close packings. The paper [H2] shows how to resolve the degeneracies by taking a small perturbation of the packing. In general, the Delaunay decomposition will depend on this perturbation. We refer to the centers of the packing as vertices, since the structure of the simplicial decomposition of space is our primary concern. For a proof that the Delaunay decomposition is a dissection of space into simplices, we refer the reader to $[R]$.

The Delaunay decomposition is dual to the well-known Voronoi decomposition. If the vertices of the Delaunay simplices are in nondegenerate position, two vertices are joined by an edge exactly when the two corresponding Voronoi cells share a face, three vertices form a face exactly when the three Voronoi cells share an edge, and four vertices form a simplex exactly when the four corresponding Voronoi cells share a vertex. In other words, two vertices are joined by an edge if they lie on a sphere that does not contain any other of the vertices, and so forth (again assuming the vertices to be in nondegenerate position). The collection of all simplices that share a given vertex is called a Delaunay star. (This is a provisional definition: it is refined below.)

Every Delaunay simplex has edges between 2 and 4 in length and, because of the saturation of the packing, a circumradius of at most 2 . We assume that every simplex $S$ in this paper comes with a fixed order on its edges, $1, \ldots, 6$. The order on the edges is to be arranged so that the first, second, and third edges meet at a vertex. We may also assume that the edges numbered $i$ and $i+3$ are opposite edges for $i=1,2,3$. We define $S\left(y_{1}, \ldots, y_{6}\right)$ to be the (ordered) simplex whose $i$ th edge has length $y_{i}$. If $S$ is a Delaunay simplex in a fixed Delaunay star, then it has a distinguished vertex, the vertex common to all simplices in the star. In this situation we assume that the edges are numbered so that the first, second, and third edges meet at the distinguished vertex.

A function, known as the compression $\Gamma(S)$, is defined on the space of all Delaunay simplices. Let $\delta_{o c t}=(-3 \pi+12 \arccos (1 / \sqrt{3})) / \sqrt{8} \approx 0.720903$ be the density of a regular octahedron with edges of length 2 . That is, place a unit ball at each vertex of the octahedron, and let $\delta_{o c t}$ be the ratio of the volume of the part of the balls in the octahedron to the volume of the octahedron. Let $S$ be a Delaunay simplex. Let $B$ be the union of 
four unit balls placed at each of the vertices of $S$. Define the compression as

$$
\Gamma(S)=-\delta_{o c t} \operatorname{vol}(S)+\operatorname{vol}(S \cap B) .
$$

We extend the definition of compression to Delaunay stars $D^{*}$ by setting $\Gamma\left(D^{*}\right)=$ $\sum \Gamma(S)$, with the sum running over all the Delaunay simplices in the star.

In this and subsequent work, we single out for special treatment the edges of length between 2 and 2.51. The constant 2.51 was determined experimentally to have a number of desirable properties. This constant appears throughout the paper. We call vertices that come within 2.51 of each other close neighbors.

We say that the convex hull of four vertices is a quasi-regular tetrahedron (or simply a tetrahedron) if all four vertices are close neighbors of one another. Suppose that we have a configuration of six vertices in bijection with the vertices of an octahedron with the property that two vertices are close neighbors if and only if the corresponding vertices of the octahedron are adjacent. Suppose further that exactly one of the three diagonals has length at most $2 \sqrt{2}$. In this case we call the convex hull of the six vertices a quasi-regular octahedron (or simply an octahedron).

The compatibility of quasi-regular tetrahedra and octahedra with the Delaunay decomposition is established in Section 3. We think of Euclidean space as the union of quasi-regular tetrahedra, octahedra, and various less-interesting Delaunay simplices. From now on, a Delaunay star is to be the collection of all quasi-regular tetrahedra, octahedra, and Delaunay simplices that share a common vertex $v$. This collection of Delaunay simplices and quasi-regular solids is often, but not always, the same as the objects called Delaunay stars in [H2]. We warn the reader of this shift in terminology.

It is convenient to measure the compression in multiples of the compression of the regular simplex of edge length 2 . We define a point (abbreviated $p t$ ) to be $\Gamma(S(2,2,2,2,2,2))$. We have $p t=11 \pi / 3-12 \arccos (1 / \sqrt{3}) \approx 0.0553736$.

One of the main purposes of this paper and its sequel is to replace the compression by a function (called the score) that has better properties than the compression. Further details on the definition of score appear in Section 2. We are now able to state the main theorem of this paper.

Theorem 1. If a Delaunay star is composed entirely of quasi-regular tetrahedra, then its score is less than 8 pt.

The idea of the proof is the following. Consider the unit sphere whose center is the center of the Delaunay star $D^{*}$. The intersection of a simplex in $D^{*}$ with this unit sphere is a spherical triangle. For example, a regular tetrahedron with edges of length 2 gives a triangle on the unit sphere of arc length $\pi / 3$. The star $D^{*}$ gives a triangulation of the unit sphere. The restriction on the lengths of the edges of a quasi-regular tetrahedron constrains the triangles in the triangulation. We classify all triangulations that potentially come from a star scoring more than $8 \mathrm{pt}$. Section 5 develops a long list of properties that must be satisfied by the triangulation of a high-scoring star.

It is then necessary to classify all the triangulations that possess the properties on this list. The original classification was carried out by a computer program, which generated all potential triangulations and checked them against the list. D. J. Muder has made a 
significant improvement in the argument by giving a direct, computer-free classification. His result appears in the Appendix.

As it turns out, there is only one triangulation that satisfies all of the properties on the list. Section 7 proves that Delaunay stars with this triangulation score less than $8 p t$. This will complete the main thread of the argument.

There are a number of estimates in this paper that are established by computer. These estimates are used throughout the paper, even though their proofs are not discussed until Sections 8 and 9 . These sections may be viewed as a series of technical appendices giving explicit formulas for the compression, dihedral angles, solid angles, volumes, and other quantities that must be estimated. The final section states the inequalities and gives details about the computerized verification. There is no vicious circle here: the results of Sections 8 and 9 do not rely on anything from Sections 2-7.

There are several functions of a Delaunay simplex that are used throughout this paper. The compression $\Gamma(S)$ has been defined above. The dihedral angle $\operatorname{dih}(S)$ is defined to be the dihedral angle of the simplex $S$ along the first edge (with respect to the fixed order on the edges of $S)$. Set $\operatorname{dih}_{\min }=\operatorname{dih}(S(2,2,2.51,2,2,2.51)) \approx 0.8639$; $\operatorname{dih}_{\max }=\operatorname{dih}(S(2.51,2,2,2.51,2,2))=\arccos (-29003 / 96999) \approx 1.874444$. We will see that dih $_{\min }$ and dih $_{\max }$ are lower and upper bounds on the dihedral angles of quasiregular tetrahedra. The solid angle (measured in steradians) at the vertex joining the first, second, and third edges is denoted $\operatorname{sol}(S)$. The intersection of $S$ with the ball of unit radius centered at this vertex has volume sol $(S) / 3$ (see $[H 1,2.1]$ ). For example, $\operatorname{sol}(S(2,2,2,2,2,2)) \approx 0.55$. Let $\operatorname{rad}(S)$ be the circumradius of the simplex $S$. In Section 2 we define two other functions: $\operatorname{vor}(S)$, which is related to the volume of Voronoi cells, and the score $\sigma(S)$. Finally, let $\eta(a, b, c)$ denote the circumradius of a triangle with edges $a, b, c$. Explicit formulas for all these functions appear in Section 8 .

\section{The Program}

By proving Theorem 1, the main purpose of this paper will be achieved. Nevertheless, it might be helpful to give a series of comments about how Theorem 1 may be viewed as the solution to the first of a handful of optimization problems that would collectively provide a solution to the Kepler conjecture.

We begin with some notation and terminology. We fix a Delaunay star $D^{*}$ about a vertex $v_{0}$, which we take to be the origin, and we consider the unit sphere at $v_{0}$. Let $v_{1}$ and $v_{2}$ be vertices of $D^{*}$ such that $v_{0}, v_{1}$, and $v_{2}$ are all close neighbors of one another. We take the radial projections $p_{i}$ of $v_{i}$ to the unit sphere with center at the origin and connect the points $p_{1}$ and $p_{2}$ by a geodesic arc on the sphere. We mark all such arcs on the unit sphere. Lemma 3.10 shows that the arcs meet only at their endpoints. The closures of the connected components of the complement of these arcs are regions on the unit sphere, called the standard regions. We may remove the arcs that do not bound one of the regions. The resulting system of edges and regions is referred to as the standard decomposition of the unit sphere.

Let $C$ be the cone with vertex $v_{0}$ over one of the standard regions. The collection of the Delaunay simplices, quasi-regular tetrahedra, and quasi-regular octahedra of $D^{*}$ in $C$ (together with the distinguished vertex $v_{0}$ ) is called a standard cluster. Each Delau- 
nay simplex in $D^{*}$ belongs to a unique standard cluster. Each triangle in the standard decomposition of the unit sphere is associated with a unique quasi-regular tetrahedron, and each tetrahedron determines a triangle in the standard decomposition (Lemma 3.7). We may identify quasi-regular tetrahedra with clusters over triangular regions.

We assign a score to each standard cluster in $[\mathrm{H} 4,3]$. In this section we define the score of a quasi-regular tetrahedron and describe the properties that the score should have in general.

Let $S$ be a quasi-regular tetrahedron. It is a standard cluster in a Delaunay star with center $v_{0}$. If the circumradius of $S$ is at most 1.41 , then we define the score to be $\Gamma(S)$.

If the circumradius is greater than 1.41, then embed the simplex $S$ in Euclidean threespace. Partition Euclidean space into four infinite regions (infinite Voronoi cells) by associating with each vertex of $S$ the points of space closest to that vertex. By intersecting $S$ with each of the four regions, we partition $S$ into four pieces $\hat{S}_{0}, \hat{S}_{1}, \hat{S}_{2}$, and $\hat{S}_{3}$, corresponding to its four vertices $v_{0}, v_{1}, v_{2}$, and $v_{3}$. Let sol ${ }_{i}$ be the solid angle at the vertex $v_{i}$ of the simplex. The expression $-4 \delta_{o c t} \operatorname{vol}\left(\hat{S}_{0}\right)+4 \operatorname{sol}_{0} / 3$ is an analytic function of the lengths of the edges for simplices $S$ that contain their circumcenters. (Explicit formulas appear in Section 8.) This function may be analytically continued to a function of the lengths of the edges for simplices $S$ that do not necessarily contain their circumcenters. Let $\operatorname{vor}(S)$ be defined as the analytic continuation of $-4 \delta_{o c t} \operatorname{vol}\left(\hat{S}_{0}\right)+4 \operatorname{sol}_{0} / 3$.

In this case define the score of $S$ to be $\operatorname{vor}(S)$. Write $\sigma(S)$ for the score of a quasiregular tetrahedron. In summary, the score is

$$
\sigma(S)= \begin{cases}\Gamma(S), & \text { if the circumradius of } S \text { is at most 1.41, } \\ \operatorname{vor}(S) & \text { otherwise. }\end{cases}
$$

The quasi-regular tetrahedron $S$ appears in four Delaunay stars. In the other three Delaunay stars, the distinguished vertices will be $v_{1}, v_{2}$, and $v_{3}$, so that $S$, viewed as a standard cluster in the other Delaunay stars, will have scores $-\delta_{\text {oct }} \operatorname{vol}\left(\hat{S}_{i}\right)+\operatorname{sol}_{i} / 3$ (or their analytic continuations), for $i=1,2,3$. By definition,

$$
\Gamma(S)=\sum_{i=0}^{3}\left(-\delta_{o c t} \operatorname{vol}\left(\hat{S}_{i}\right)+\operatorname{sol}_{i} / 3\right) .
$$

The sum of the scores of $S$, for each of the four vertices of $S$, is $4 \Gamma(S)$. This is the same total that is obtained by summing the compression of $S$ at each of its vertices. This is the property we need to relate the score to the density of the packing. It means that although the score reapportions the compression among neighboring Delaunay stars, the average of the compression over a large region of space equals the average of the score over the same region, up to a negligible boundary term.

The analytic continuation in the definition of $\operatorname{vor}(S)$ has the following geometric interpretation. If the circumcenter of a quasi-regular tetrahedron $S$ is not contained in $S$, a small tip of the infinite Voronoi cell at $v_{0}$ (or some other vertex) will protrude through the opposite face of the Delaunay simplex. The volume of this small protruding tip is not counted in $-4 \delta_{o c t} \operatorname{vol}\left(\hat{S}_{0}\right)+4 \operatorname{sol}_{0} / 3$, but it is counted in the analytic continuation. The analytic continuations of the scores of $S$ for each of the other three vertices acquire a term representing the negative volume of a part of the tip. The three parts together 
constitute the entire tip, so that the negative volumes exactly offset the volume of the tip, and the sum of the four scores of $S$ is $4 \Gamma(S)$. Details appear in [H4].

The general definition of the score will have similar properties. To each standard cluster of a Delaunay star $D^{*}$ a score will be assigned. The rough idea is to let the score of a simplex in a cluster be the compression $\Gamma(S)$ is the circumradius of every face of $S$ is small, and otherwise to let the score be defined by Voronoi cells (in a way that generalizes the definition for quasi-regular tetrahedra).

The score $\sigma\left(D^{*}\right)$ of a Delaunay star is defined as the sum of the scores of its standard clusters. The score has the following properties [H4, 3.1 and 3.5]:

1. The score of a standard cluster depends only on the cluster, and not on the way it sits in a Delaunay star or in the Delaunay decomposition of space.

2. The Delaunay stars of the face-centered cubic and hexagonal close packings score exactly $8 p t$.

3. The score is asymptotic to the compression over large regions of space. We make this more precise. Let $\Lambda$ denote the vertices of a saturated packing. Let $\Lambda_{N}$ denote the vertices inside the ball of radius $N$. (Fix a center.) Let $D^{*}(v)$ denote the Delaunay star at $v \in \Lambda$. Then the score satisfies (in Landau's notation)

$$
\sum_{\Lambda_{N}} \sigma\left(D^{*}(v)\right)=\sum_{\Lambda_{N}} \Gamma\left(D^{*}(v)\right)+O\left(N^{2}\right) .
$$

Lemma 2.1. If the score of every Delaunay star in a saturated packing is at most $s<16 \pi / 3$, then the density of the packing is at most $16 \pi \delta_{\text {oct }} /(16 \pi-3 s)$. If the score of every Delaunay star in a packing is at most 8 pt, then the density of the packing is at most $\pi / \sqrt{18}$.

Proof. The second claim is the special case $s=8 p t$. The proof relies on property 3 . The number of vertices such that $D^{*}(v)$ meets the boundary of the ball $B_{N}$ of radius $N$ has order $O\left(N^{2}\right)$. Since the Delaunay stars give a fourfold cover of $\mathbb{R}^{3}$, we have

$$
\begin{aligned}
4\left(-\delta_{o c t} \operatorname{vol}\left(B_{N}\right)+\left|\Lambda_{N}\right| \frac{4 \pi}{3}\right) & =\sum_{\Lambda_{N}}\left(-\delta_{o c t} \operatorname{vol}\left(D^{*}(v)\right)+\frac{4 \operatorname{sol}\left(D^{*}(v)\right)}{3}+O\left(N^{2}\right)\right. \\
& =\sum_{\Lambda_{N}} \Gamma\left(D^{*}(v)\right)+O\left(N^{2}\right) \\
& =\sum_{\Lambda_{N}} \sigma\left(D^{*}(v)\right)+O\left(N^{2}\right) \\
& \leq s\left|\Lambda_{N}\right|+O\left(N^{2}\right) .
\end{aligned}
$$

Rearranging, we get

$$
\frac{4 \pi\left|\Lambda_{N}\right|}{3 \operatorname{vol}\left(B_{N}\right)} \leq \frac{\delta_{o c t}}{(1-3 s / 16 \pi)}+\frac{O\left(N^{2}\right)}{\operatorname{vol}\left(B_{N}\right)} .
$$

In the limit the left-hand side is the density and the right-hand side is the bound. Similar arguments can be found in [H1] and [H2]. 
The following conjecture is fundamental. By the lemma, this conjecture implies the Kepler conjecture. The lemma also shows that weaker bounds than $8 p t$ on the score might be used to give new upper bounds on the density of sphere packings.

Conjecture 2.2. The score of every Delaunay star is at most 8 pt.

The basic philosophy behind the approach of this paper is that quasi-regular tetrahedra are the only clusters that give a positive score, standard clusters over quadrilateral regions should be the only other clusters that may give a score of zero, and every other standard cluster should give a negative score. Moreover, we will prove that no quasi-regular tetrahedron gives more than $1 \mathrm{pt}$.

Thus, heuristically, we try to obtain a high score by including as many triangular regions as possible. If we allow any other shape, preference should be given to quadrilaterals. Any other shape of region should be avoided if possible. If these other regions occur, they should be accompanied by additional triangular regions to compensate for the negative score of the region. We will see later in this paper that even triangular regions tend to give a low score unless they are arranged to give five triangles around each vertex.

The main steps in a proof of the Kepler conjecture are

1. A proof that even if all regions are triangular the total score is less than $8 \mathrm{pt}$.

2. A proof that standard clusters in regions of more than three sides score at most 0 pt.

3. A proof that if all of the standard regions are triangles or quadrilaterals, then the total score is less than $8 \mathrm{pt}$ (excluding the case of pentagonal prisms).

4. A proof that if some standard region has more than four sides, then the star scores less than 8 pt.

5. A proof that pentagonal prisms score less than $8 \mathrm{pt}$.

The division of the problem into these steps is quite arbitrary. They were originally intended to be steps of roughly equal magnitude, although is has turned out that a construction in [H4] has made the second step substantially easier than the long calculations of the third step.

This paper carries out the first step. The second step of the program is also complete [H4]. Partial results are known for the third step [H5]. In the fourth step it will be necessary to argue that these regions take up too much space, give too little in return, and have such strongly incompatible shapes that they cannot be part of a winning strategy.

To make step 5 precise, we define pentagonal prisms to be Delaunay stars whose standard decomposition has ten triangles and five quadrilaterals, with the five quadrilaterals in a band around the equator, capped on both ends by five triangles (Diagram 2.3). The conjecture in this section asserts, in particular, that pentagonal prisms, which created such difficulties in [H2], score less than $8 \mathrm{pt}$. The final step has been separated from the third step, because the estimates are expected to be more delicate for pentagonal prisms than for a general Delaunay star in the third step.

One of the main shortcomings of the compression is that pentagonal prisms have compression greater than $8 \mathrm{pt}$ (see [H2]). Numerical evidence suggests that the upper bound on the compression is attained by a pentagonal prism, denoted $D_{\mathrm{ppdp}}^{*}$ in [H2], at 


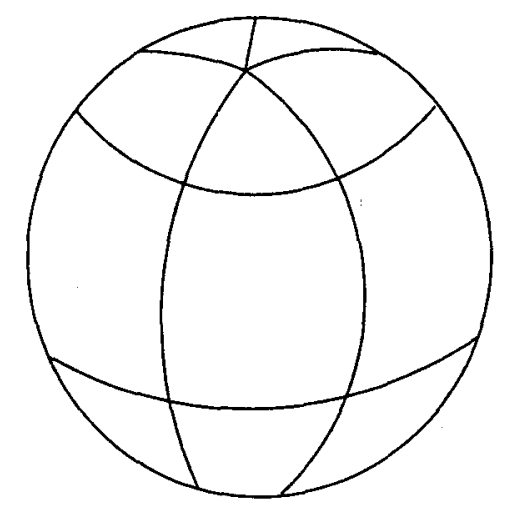

Diagram 2.3

about $8.156 \mathrm{pt}$, and this means that the link between the compression and the Kepler conjecture is indirect. The score appears to correct this shortcoming.

What evidence is there for the conjecture and program? They come as the result of extensive computer experimentation. I have checked the conjecture against much of the data obtained in the numerical studies of $[\mathrm{H} 2,9.3]$. The data suggest that the score tends to give a dramatic improvement over the compression, often improving the bound by more than a point. The score of the particularly troublesome pentagonal prism $D_{\mathrm{ppdp}}^{*}$ drops safely under $8 \mathrm{pt}$. I have checked a broad assortment of other pentagonal prisms and have found them all to score less than $8 p t$.

The second step shows that no serious pathologies can arise. The only way to form a Delaunay star with a positive score is by arranging a number of quasi-regular tetrahedra around a vertex (together with other standard clusters than can only lower the score). There must be at least eight tetrahedra to score $8 p t$, and if there are any distortions in these tetrahedra, there must be at least nine. However, as this paper shows, too many quasi-regular tetrahedra in any star are also harmful. Future papers will impose additional limits on the structure of the optimal Delaunay star.

\section{Quasi-Regular Tetrahedra}

This section studies the compatibility of the Delaunay simplices and the quasi-regular solids. Fix three vertices $v_{1}, v_{2}$, and $v_{3}$ that are close rieighbors to one another. Let $T$ be the triangle with vertices $v_{i}$. It does not follow that $T$ is the face of a Delaunay simplex. However, as we will see, when $T$ is not the face of a simplex, the arrangement of the surrounding simplices is almost completely determined.

If $T$ is not the face of a Delaunay simplex, then we will show that there are two additional vertices $v_{0}$ and $v_{0}^{\prime}$, where $v_{0}$ and $v_{0}^{\prime}$ are close neighbors to $v_{1}, v_{2}$, and $v_{3}$. This means that there are two quasi-regular tetrahedra $S_{1}$ and $S_{2}$ with vertices $\left(v_{0}, v_{1}, v_{2}, v_{3}\right)$ and $\left(v_{0}^{\prime}, v_{1}, v_{2}, v_{3}\right)$, respectively, that have the common face $T$ (see Diagram 3.1(a)). We see that $S_{1} \cup S_{2}$ is the union of three Delaunay simplices with vertices $\left(v_{0}, v_{0}^{\prime}, v_{1}, v_{2}\right)$, $\left(v_{0}, v_{0}^{\prime}, v_{2}, v_{3}\right)$, and $\left(v_{0}, v_{0}^{\prime}, v_{3}, v_{1}\right)$ in Diagram 3.1(b). This section establishes that this 
is the only situation in which quasi-regular tetrahedra are not Delaunay simplices: they must come in pairs and their union must be three Delaunay simplices joined along a common edge. The decomposition of this paper is obtained by taking each such triple of Delaunay simplices (3.1(b)) and replacing the triple by a pair of quasi-regular tetrahedra (3.1(a)).
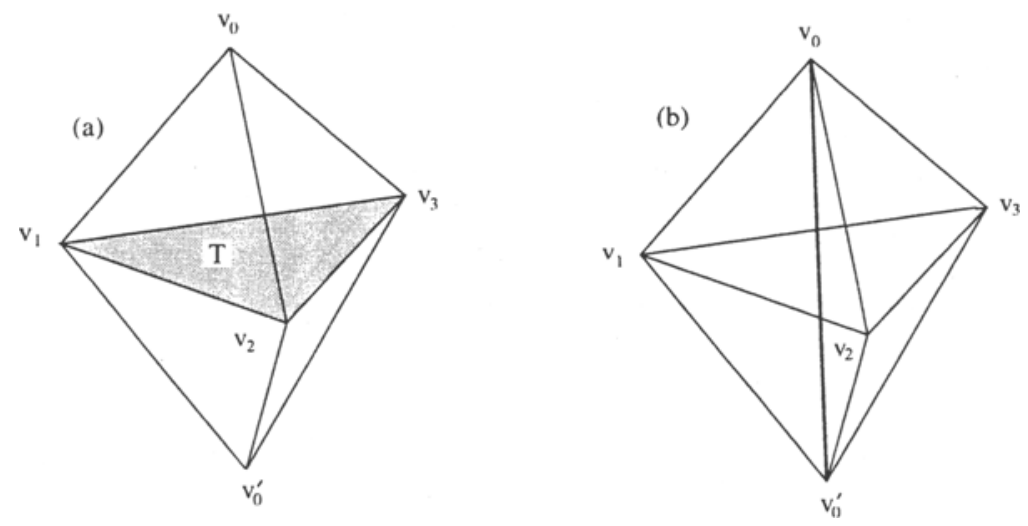

Diagram 3.1

From the dual perspective of Voronoi cells, the Voronoi cell at $v_{0}$ (or $v_{0}^{\prime}$ ) would have a small tip protruding from $S_{1}$ through $T$, if the vertex $v_{0}^{\prime}$ were not present. The vertex $v_{0}^{\prime}$ slices off this protruding tip so that the Voronoi cells at $v_{0}$ and $v_{0}^{\prime}$ have a small face in common.

Lemma 3.2. Suppose that the circumradius of the triangle $T$ is less that $\sqrt{2}$. Then $T$ is the face of a Delaunay simplex.

Proof. Let $r<\sqrt{2}$ be the radius of the circle that circumscribes $T$, and let $c$ be the center of the circle. The sphere of radius $r$ at $c$ does not contain any vertices of $D^{*}$ other than $v_{1}, v_{2}$, and $v_{3}$. By the definition of the Delaunay decomposition (as described in the Introduction), this implies that $T$ is the face of a simplex.

Remark 3.3. We have several constraints on the edge lengths, if $T$ is not a face of a Delaunay simplex. Consider the circumradius $\eta(a, b, c)$ of a triangle whose edges have lengths $a, b, c$ between 2 and 2.51 . Since $2.51^{2}<2^{2}+2^{2}$, we see that the triangle is acute, so that $\eta(a, b, c$,$) is monotonically increasing in a, b$, and $c$. This gives simple estimates relating the circumradius to $a, b$, and $c$. The circumradius is at most $\eta(2.51,2.51,2.51)=$ $2.51 / \sqrt{3} \approx 1.449$. If the circumradius is at least $\sqrt{2} \approx 1.41421$, then $a, b$, and $c$ are greater than $2.3(\eta(2.3,2.51,2.51) \approx 1.41191<\sqrt{2})$. Under the same hypothesis, two of $a, b$, and $c$ are greater than $2.41(\eta(2.41,2.41,2.51)<\sqrt{2})$. Finally, at least one edge has length greater than $2.44(\eta(2.44,2.44,2.44)<\sqrt{2})$.

Lemma 3.4. Let $T$ be the triangle with vertices $v_{i}$. Assume that the vertices $v_{i}$ are close neighbors of one another. Suppose there is a vertex $v_{0}$ that lies closer to the circumcenter 
of $T$ than the vertices of $T$ do. Then the vertex $v_{0}$ satisfies $2 \leq\left|v_{0}-v_{i}\right|<2.15$, for $i=1,2,3$. In particular, the convex hull of $v_{0}, \ldots, v_{3}$ is a quasi-regular tetrahedron $S$ with face $T$.

Another way of stating the hypothesis on the circumcenter is to say that the plane of $T$ separates $v_{0}$ from the circumcenter of $S$. Because of the constraints on the edge lengths in Remark 3.3, the three other faces of $S$ are faces of Delaunay simplices.

Proof. We defer the proof to Section 8.2.5.

Lemma 3.5. Let $v, v_{1}, v_{2}, v_{3}$, and $v_{4}$ be distinct vertices with pairwise distances at least 2. Suppose that the pairs $\left(v_{i}, v_{j}\right)$ are close neighbors for $\{i, j\} \neq\{1,4\}$. Then $v$ does not lie in the convex hull of $\left(v_{1}, v_{2}, v_{3}, v_{4}\right)$.

Proof. For a contradiction, suppose $v$ lies in the convex hull. Since $\left|v-v_{i}\right| \geq 2$ and for $\{i, j\} \neq\{1,4\}, v_{i}$ and $v_{j}$ are close neighbors, the angle formed by $v_{i}$ and $v_{j}$ at the vertex $v$ is at most $\theta_{0}=\arccos \left(1-2.51^{2} / 8\right) \approx 1.357$. Each such pair of vertices gives a geodesic arc of length at most $\theta_{0}$ radians on the unit sphere centered at $v$. We obtain in this way a triangulation of the unit sphere by four triangles, two with edges of length at most $\theta_{0}=2 \arcsin (2.51 / 4)$ radians, and two others that fit together to form a quadrilateral with edges of at most $\theta_{0}$ radians. By the spherical law of cosines, the area formula for a spherical triangle, and [H2, 6.1], each of the first two triangles has area at most $3 \arccos \left(\cos \theta_{0} /\left(1+\cos \theta_{0}\right)\right)-\pi \approx 1.04$. By the same lemma, the quadrilateral has area at most the area of a regular quadrilateral of side $\theta_{0}$, or about 2.8. Since the combined area of the two triangles and quadrilateral is less than $4 \pi$, they cannot give the desired triangulation. To see that $v$ cannot lie on the boundary, it is enough to check that a triangle having two edges of lengths between 2 and 2.51 cannot contain a point that has distance 2 or more from each vertex. We leave this as an exercise.

Corollary 3.5.1. No vertex of the packing is ever an interior point of a quasi-regular tetrahedron or octahedron.

Proof. The corollary is immediate for a tetrahedron. For an octahedron, draw the distinguished diagonal and apply the lemma to each of the four resulting simplices.

Let $T$ be a triangle of circumradius between $\sqrt{2}$ and $2.51 / \sqrt{3}$, with edges of length between 2 and 2.51. Consider the line through the circumcenter of $T$, perpendicular to the plane of $T$. Let $s$ be the finite segment in this line whose endpoints are the circumcenters of the two simplices with face $T$ formed by placing an additional vertex at distance 2 from the three vertices of $T$ on either side of the plane through $T$.

Lemma 3.6. Let $S$ be a simplex formed by the vertices of $T$ and a fourth vertex $v_{0}^{\prime}$. Suppose that the circumcenter of $S$ lies on the segment $s$. Assume that $v_{0}^{\prime}$ has distance at least 2 from each of the vertices of $T$. Then $v_{0}^{\prime}$ has distance less than 2.3 from each of the vertices of $T$. 
Proof. Let $v_{1}, v_{2}, v_{3}$ be the vertices of $T$. For a contradiction, assume that $v_{0}^{\prime}$ has distance at least 2.3 from a vertex $v_{1}$ of $T$. Lemma 3.4 shows that the plane through $T$ does not separate $v_{0}^{\prime}$ from the circumcenter of $S$. If the circumcenter lies in $s$ (and is not separated from $v_{0}^{\prime}$ by the plane through $T$ ), then by moving $v_{0}^{\prime}$ to decrease the circumradius, the circumcenter remains in $s$.

Let $S$ be the simplex with vertices $v_{0}^{\prime}, v_{1}, v_{2}$, and $v_{3}$. The circumcenter of $S$ lies in the interior of $S$. We omit the proof, because it is established by methods similar to (but longer than) the proof of Lemma 3.4. Thus, the circumradius is increasing in the lengths of $\left|v_{0}^{\prime}-v_{i}\right|$, for $i=1,2,3$ (see paragraph 8.2.4).

Let $R$ be the circumradius of a simplex with face $T$ and center an endpoint of $s$. We will prove that the circumradius of $S$ is greater than $R$, contrary to our hypothesis. Moving $v_{0}^{\prime}$ to decrease the circumradius, we may take the distances to $v_{i}$ to be precisely $2.3,2$, and 2 . We may move $v_{1}, v_{2}$, and $v_{3}$ along their fixed circumscribing circle until $\left|v_{2}-v_{3}\right|=2.51$ and $\left|v_{1}-v_{2}\right|=\left|v_{1}-v_{3}\right|$ in a way that does not decrease any of the distances from $v_{0}^{\prime}$ to $v_{i}$. Repeating the previous step, we may retain our assumption that $v_{0}^{\prime}$ has distances exactly $2.3,2$, and 2 from the vertices $v_{i}$ as before. We have reduced the problem to a one-dimensional family of tetrahedra parametrized by the radius $r$ of the circumscribing circle of $T$. Set $x(r)=\left|v_{1}-v_{3}\right|=\left|v_{2}-v_{3}\right|$. To obtain our desired contradiction, we must show that the circumradius $R^{\prime}(r)$ of the simplex $S(2.3,2,2,2.51, x(r), x(r))$ satisfies $R^{\prime}(r)>R(r)$. Since both $R^{\prime}$ and $R$ are increasing in $r$, for $r \in[\sqrt{2}, 2.51 / \sqrt{3}]$, the desired inequality follows if we evaluate the 200 constants

$$
R^{\prime}\left(r_{i}\right)-R\left(r_{i+1}\right) \quad \text { for } \quad r_{i}=\sqrt{2}\left(\frac{2.51}{\sqrt{3}}-\sqrt{2}\right) \frac{i}{200},
$$

for $i=0, \ldots, 199$, and check that they are all positive. (The smallest is about 0.00005799 , which occurs for $i=199$.)

Let $T$ be a triangle made up of three close neighbors. Suppose that $T$ is not the face of a Delaunay simplex. There exists a vertex $v_{0}$ whose distance to the circumcenter of $T$ is less than the circumradius of $T$. Let $S$ be the quasi-regular tetrahedron formed by $v_{0}$ and the vertices of $T$. It is not a Delaunay simplex, so there exists a vertex $v_{0}^{\prime}$ that is less than the circumradius of $S$ from the circumcenter of $S$. Let $S^{\prime}$ be the simplex formed by $v_{0}^{\prime}$ and the vertices of $T$. It is not a Delaunay simplex either. The circumcenter of $S$ lies in the segment $s$ of Lemma 3.4, so the circumcenter of $S^{\prime}$ does too. The lengths of the edges of $S$ and $S^{\prime}$ other than $T$ are constrained by Lemmas 3.4 and 3.6. In particular, in light of Remark 3.3, the faces other than $T$ of $S$ and $S^{\prime}$ are faces of Delaunay simplices.

If $v_{0}$ and $v_{0}^{\prime}$ lie on the same side of the plane through $T$, then either $v_{0}^{\prime}$ lies in $S, v_{0}$ lies in $S^{\prime}$, or the faces of $S$ and $S^{\prime}$ intersect nonsimplicially. None of these situations can occur because a nondegenerate Delaunay decomposition is a Euclidean simplicial complex and because of Lemma 3.5. We conclude that $v_{0}$ and $v_{0}^{\prime}$ lie on opposite sides of the plane through $T$.

$S \cup S^{\prime}$ is bounded by Delaunay faces, so $S \cup S^{\prime}$ is a union of Delaunay simplices. The fourth vertex of the Delaunay simplex in $S \cup S^{\prime}$ with face $\left(v_{0}, v_{1}, v_{2}\right)$ cannot be $v_{3}$ ( $S$ is not a Delaunay simplex), so it must be $v_{0}^{\prime}$. Similarly, $\left(v_{0}, v_{0}^{\prime}, v_{2}, v_{3}\right)$ and $\left(v_{0}, v_{0}^{\prime}, v_{3}, v_{1}\right)$ are Delaunay simplices. These three Delaunay simplices cannot be quasi-regular tetrahedra by Lemma 8.3.2. 
The assumption that $T$ is not a face has completely determined the surrounding geometry: there are two quasi-regular tetrahedra $S$ and $S^{\prime}$ along $T$ such that $S \cup S^{\prime}$ is a union of three Delaunay simplices.

Lemma 3.7. Let $L$ be a union of standard regions. Suppose that the boundary of $L$ consists of three edges. Then either $L$ or its complement is a single triangle.

For example, the interior of $L$ cannot have the form of Diagram 3.8. Lemma 3.5 (proof) shows that if all regions are triangles, then there are at least 12 triangles, so that the exterior of $L$ cannot have the form of Diagram 3.8 either.

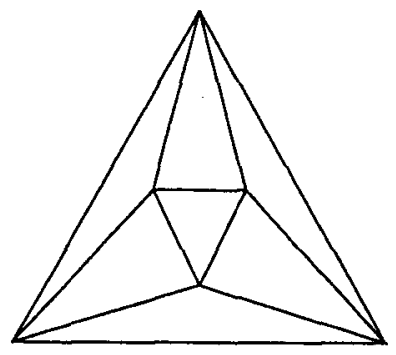

Diagram 3.8

Proof. Replacing $L$ by its complement if necessary, we may assume that the area of $L$ is less than its complement. The triangular boundary corresponds to four vertices $v_{0}$ (the origin), $v_{1}, v_{2}$, and $v_{3}$. The close-neighbor constraints on the lengths show that the convex hull of $v_{0}, \ldots, v_{3}$ is a quasi-regular tetrahedron. By construction each quasiregular tetrahedron is a single cluster.

We say that a point $v \in \mathbb{R}^{3}$ is enclosed by a region on the unit sphere is the interior of the cone (with vertex $v_{0}$ ) over that region contains $v$. For example, in Diagram 3.9, the point $v$ is enclosed by the given spherical triangle.

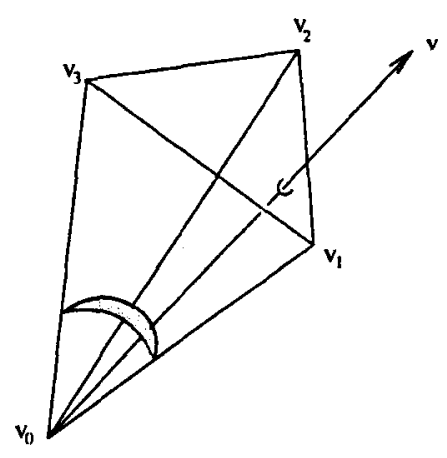

Diagram 3.9

The following lemma was used in Section 2 to define the standard decomposition. 
Lemma 3.10. Fix a Delaunay star $D^{*}$ with center $v_{0}$. Draw geodesic arcs on the unit sphere at $v_{0}$ for every triple of close neighbors $v_{0}, v_{1}, v_{2}$ (as in Section 2). The resulting system of arcs do not meet except at endpoints.

Proof. Our proof is based on the fact that a nondegenerate Delaunay decomposition is a Euclidean simplicial complex. Let $T_{1}$ and $T_{2}$ be two triangles made from two such triples of close neighbors. We have $T_{i} \subset S_{i} \cup S_{i}^{\prime}$, where $S_{i}$ and $S_{i}$ are the Delaunay simplices with face $T_{i}$ if $T_{i}$ is the face of a Delaunay simplex, and they are the two quasi-regular tetrahedra with face $T_{i}$ constructed above, otherwise. Since a nondegenerate Delaunay decomposition is a Euclidean simplicial complex, $S_{1} \cup S_{1}^{\prime}$ meets $S_{2} \cup S_{2}^{\prime}$ simplicially. By the restrictions on the lengths of the edges in Lemmas 3.4 and 3.6, this forces $T_{1}$ to intersect $T_{2}$ simplicially. The result follows.

\section{Quadrilaterals}

Fix a Delaunay star composed entirely of quasi-regular tetrahedra and consider the associated triangulation of the unit sphere. Let $L$ be a region of the sphere bounded by four edges of the triangulation. $L$ will be the union of two or more triangles. Replacing $L$ by its complement in the unit sphere if necessary, we assume that the area of $L$ is less than that of its complement.

We claim that, in this context, $L$ is the union of either two or four triangles, as illustrated in Diagram 4.1. In particular, $L$ encloses at most one vertex. If a diagonal to the quadrilateral $L$ is an edge of the triangulation, the region $L$ is divided into two triangles each associated with a quasi-regular tetrahedron. In particular, there is no enclosed vertex. (Lemma 3.7 precludes any subtriangulation of a triangular region.) If, however, there is a single enclosed vertex and neither diagonal is an edge of the triangulation, then the only possible triangulation of $L$ is the one of the diagram. Proposition 4.2 completes the proof of the claim.
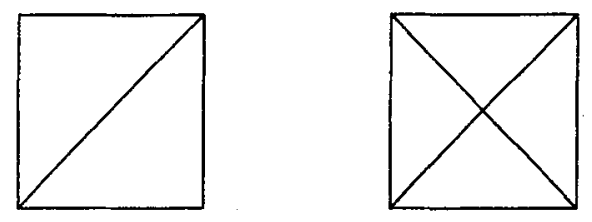

Diagram 4.1

Proposition 4.2. A union of regions (of area less than $2 \pi$ ) bounded by exactly four edges cannot enclose two vertices of distance at most 2.51 from the origin.

This argument is somewhat delicate: if our parameter 2.51 had been set at 2.541 , for instance, such an arrangement would exist. First we prove a useful reduction. 
Lemma 4.3. Assume a figure exists with vectors $v_{1}, \ldots, v_{4}, v$, and $v^{\prime}$ subject to the constraints

$$
\begin{aligned}
2 & \leq\left|v_{i}\right| \leq 2.51, \\
2 & \leq\left|v_{i}-v_{i+1}\right| \leq k_{i}, \\
2 & \leq\left|v_{i}-v_{i+2}\right|, \\
2 & \leq\left|v-v^{\prime}\right|, \\
h_{i} & \leq\left|w-v_{i}\right|, \\
2 & \leq|w| \leq \ell \quad \text { for } w=v, v^{\prime} \text { and } i=1, \ldots, 4 \quad(\bmod 4),
\end{aligned}
$$

where $\ell, h_{i}$, and $k_{i}$ are fixed constants that satisfy $\ell \in[2.51,2 \sqrt{2}], h_{i} \in[2,2 \sqrt{2})$, $k_{i} \in[2,2.51]$. Let $L$ be the quadrilateral on the unit sphere with vertices $v_{i} /\left|v_{i}\right|$ and edges running between consecutive vertices. Assume that $v$ and $v^{\prime}$ lie in the cone at the origin obtained by scaling $L$. Then another figure exists made of $a(n e w)$ collection of vectors $v_{1}, \ldots, v_{4}, v$, and $v^{\prime}$ subject to the constraints above together with the additional constraints

$$
\begin{aligned}
\left|v_{i}-v_{i+1}\right| & =k_{i}, \\
\left|v_{i}\right| & =2 \quad \text { for } \quad i=1, \ldots, 4, \\
|v| & =\left|v^{\prime}\right|=\ell .
\end{aligned}
$$

Moreover, the quadrilateral $L$ may be assumed to be convex.

Proof. By rescaling $v$ and $v^{\prime}$, we may assume that $|v|=\left|v^{\prime}\right|=\ell$. (Moving $v$ or $v^{\prime}$ away from the origin increases its distance from the other vertices of the configuration.)

The diagonals satisfy $\left|v_{2}-v_{4}\right|>2.1$ and $\left|v_{1}-v_{3}\right|>2.1$. Otherwise, if say $\left|v_{1}-v_{3}\right| \leq$ 2.1 , then the faces with vertices $\left(v_{1}, v_{2}, v_{3}\right)$ and $\left(v_{1}, v_{4}, v_{3}\right)$ have circumradius less than $\sqrt{2}$. The edge from 0 to $v$ has length at most $\sqrt{2}$, so this edge cannot intersect these faces by the Euclidean simplicial complex argument used before Lemma 3.7 and in Lemma 3.10. By Lemma 3.5, $v$ cannot lie in the convex hull of $\left(0, v_{1}, v_{3}, v_{i}\right)$. This leaves $v$ nowhere to go, and a figure with $\left|v_{1}-v_{3}\right| \leq 2.1$ does not exist.

Next, we claim that we may assume that the quadrilateral $L$ is convex (in the sense that it contains the geodesic arcs between any two points in the region). To see this, suppose the vertex $v_{i}$ lies in the cone over the convex hull of the other three vertices $v_{j}$. Consider the plane $P$ through the origin, $v_{i-1}$, and $v_{i+1}$. The reflection $v_{i}^{\prime}$ of $v_{i}$ through $P$ is no closer to $v, v^{\prime}$, or $v_{i+2}$ and has the same distance to the origin, $v_{i-1}$, and $v_{i+1}$. Thus, replacing $v_{i}$ with $v_{i}^{\prime}$ if necessary, we may assume that $L$ is convex.

Most of the remaining deformations are described as pivots. We fix an axis and rotate a vertex around a circle centered along and perpendicular to the given axis. If, for example, $\left|v_{3}-v_{4}\right|<k_{3}$, we pivot the vertex $v_{4}$ around the axis through 0 and $v_{1}$ until $\left|v_{3}-v_{4}\right|=k_{3}$. It follows from the choice of axes that the distances from $v_{4}$ to the origin and $v_{1}$ are left unchanged, and it follows from the convexity of $L$ that $\left|w-v_{4}\right|$ increases for $w=v_{2}, v_{3}, v$, and $v^{\prime}$. Similarly, we may pivot vertices $v_{i}$ along the axis through the origin and $v_{i+1}$ until $\left|v_{i}-v_{i-1}\right|=k_{i}$, for all $i$.

Fix an axis through two opposite vertices (say $v_{1}$ and $v_{3}$ ) and pivot another vertex (say $v_{2}$ ) around the axis toward the origin. We wish to continue by picking different axes 
and pivoting until $\left|v_{i}\right|=2$, for $i=1,2,3,4$. However, this process appears to break down in the event that a vertex $v_{i}$ has distance $h_{i}$ from one of the enclosed vertices and the pivot toward the origin moves $v_{i}$ closer to that enclosed vector. We must check that this situation can be avoided.

Interchanging the roles of $\left(v_{1}, v_{3}\right)$ with $\left(v_{2}, v_{4}\right)$ as necessary, we continue to pivot until $\left|v_{1}\right|=\left|v_{3}\right|=2$ or $\left|v_{2}\right|=\left|v_{4}\right|=2$ (say the former). We claim that either $v_{2}$ has distance greater than $h_{2}$ from $v$ or that pivoting $v_{2}$ around the axis through $v_{1}$ and $v_{3}$ moves $v_{2}$ away from $v$. If not, we find that $\left|v_{1}\right|=\left|v_{3}\right|=2,\left|v-v_{2}\right|=h_{2}$ and that $v$ lies in the cone $C=C\left(v_{2}\right)$ with vertex $v_{2}$ spanned by the vectors from $v_{2}$ to the origin, $v_{1}$, and $v_{3}$. (This relies on the convexity of the region $L$.)

To complete the proof, we show that this figure made from $\left(0, v_{1}, v_{2}, v_{3}, v\right)$ cannot exist. Contract the edge $\left(v, v_{2}\right)$ as much as possible keeping the triangle $\left(0, v_{1}, v_{3}\right)$ fixed, subject to the constraints that $v \in C\left(v_{2}\right)$ and $\left|w-w^{\prime}\right| \geq 2$, for $w=v, v_{2}$ and $w^{\prime}=0$, $v_{1}, v_{3}$. This contraction gives $\left|v-v_{2}\right| \leq h_{2}<2 \sqrt{2}$.

Case 1: $v$ lies in the plane of $\left(0, v_{2}, v_{3}\right)$. This gives an impossibility: crossing edges $\left(v, v_{2}\right),\left(0, v_{3}\right)$ of length less than $2 \sqrt{2}$. Similarly, $v$ cannot lie in the plane of $\left(0, v_{1}, v_{2}\right)$.

Case 2: $v$ lies in the interior of the cone $C\left(v_{2}\right)$. The contraction gives $\left|v-w^{\prime}\right|=2$ for $w=v, v_{2}$ and $w^{\prime}=0, v_{1}, v_{3}$. The edge $\left(v, v_{2}\right)$ divides the convex hull of $\left(0, v_{1}, v_{2}, v_{3}, v\right)$ into three simplices. Consider the dihedral angles of these simplices along this edge. The dihedral angle of the simplex $\left(v_{1}, v_{2}, v_{3}, v\right)$ is less than $\pi$. The dihedral angles of the other two are less than $\operatorname{dih}(S(2 \sqrt{2}, 2,2,2,2,2))=\pi / 2$. Hence, the dihedral angles along the diagonal cannot sum to $2 \pi$ and the figure does not exist.

Case 3: $v$ lies in the plane of $\left(v_{2}, v_{1}, v_{3}\right)$. Let $r$ be the radius of the circle in this plane passing through $v_{1}$ and $v_{3}$ obtained by intersecting the plane with a sphere of radius 2 at the origin. We have $2 r \geq\left|v_{1}-v_{3}\right|>2 \sqrt{2}$ because otherwise we have the impossible situation of crossing edges $\left(v_{1}, v_{3}\right)$ and $\left(v_{2}, v\right)$ of length less than $2 \sqrt{2}$. Let $H$ be the perpendicular bisector of the segment $\left(v_{1}, v_{3}\right)$. By reflecting $v$ through $H$ if necessary we may assume that $v$ and $v_{2}$ lie on the same side of $H$, say the side of $v_{3}$. Furthermore, by contracting $\left(v, v_{2}\right)$, we may assume without loss of generality that $\left|v_{3}-v\right|=\mid v_{2}-$ $v_{3} \mid=2$. Let $f(r)=\left|v-v_{2}\right|$, as a function of $r . f$ is increasing in $r$. The inequalities $2 \sqrt{2}>h_{2} \geq\left|v-v_{2}\right| \geq f(\sqrt{2})=2 \sqrt{2}$ give the desired contradiction.

Proof of Proposition 4.2. Assume for a contradiction that $v$ and $v^{\prime}$ are vertices enclosed by $L$. Let the center of the Delaunay star be at the origin, and let $v_{1}, \ldots, v_{4}$, indexed consecutively, be the four vertices of the Delaunay star that determine the extreme points of $L$.

We describe a sequence of deformations of the configuration (formed by the vertices $\left.v_{1}, \ldots, v_{4}, v, v^{\prime}\right)$ that transform the original configuration of vertices into particular rigid arrangements below. We show that these rigid arrangements cannot exist, and from this it follows that the original configuration does not exist either. These deformations preserve the constraints of the problem. To be explicit, we assume that $2 \leq|w| \leq 2.51$, that $2 \leq\left|w-v_{i}\right|$ if $w \neq v_{i}$, that $2 \leq\left|v-v^{\prime}\right|$, and that $\left|v_{i}-v_{i+1}\right| \leq 2.51$, for $i=1,2,3,4$ and $w=v_{1}, \ldots, v_{4}, v, v^{\prime}$. Here and elsewhere we take our subscripts modulo 4 , so that 
$v_{1}=v_{5}$, and so forth. The deformations also keep $v$ and $v^{\prime}$ in the cone at the origin that is determined by the vertices $v_{i}$.

We consider some deformations that increase $\left|v-v^{\prime}\right|$. By Lemma 4.3, we may assume that $\left|v_{i}\right|=2,|v|=\left|v^{\prime}\right|=\left|v_{i}-v_{i+1}\right|=2.51$, for $i=1,2,3,4$. If, for some $i$, we have $\left|v_{i}-v\right|>2$ and $\left|v_{i}-v^{\prime}\right|>2$, then we fix $v_{i-1}$ and $v_{i+2}$ and pivot $v_{i+1}$ around the axis through the origin and $v_{i+2}$ away from $v$ and $v^{\prime}$. The constraints $\left|v_{i+1}-v_{i}\right|=\left|v_{i}-v_{i-1}\right|=2.51$ force us to drag $v_{i}$ to a new position on the sphere of radius 2 . By making this pivot sufficiently small, we may assume that $\left|v_{i}-w\right|$ and $\left|v_{i+1}-w\right|$, for $w=v, v^{\prime}$, are greater than 2 .

The vertices $v$ and $v^{\prime}$ cannot both have distance 2 from both $v_{i+2}$ and $v_{i-1}$, for then we would have $v=v^{\prime}$. So one of them, say $v$, has distance exactly 2 from at most one of $v_{i+2}$ and $v_{i-1}$ (say $v_{i+2}$ ). Thus, $v$ may be pivoted around the axis through the origin and $v_{i+2}$ away from $v^{\prime}$. In this way, we increase $\left|v-v^{\prime}\right|$ until $\left|v_{i}-v\right|=2$ or $\left|v_{i}-v^{\prime}\right|=2$, for $i=1,2,3,4$.

Suppose one of $v, v^{\prime}$ (say $v$ ) has distance 2 from $v_{i}, v_{i+1}$, and $v_{i+2}$. The configuration is completely rigid. By symmetry, the vertices $v_{i-1}$ and $v^{\prime}$ must be the reflections of $v_{i+1}$ and $v$, respectively, through the plane through $0, v_{i}$, and $v_{i+2}$. In particular, $v^{\prime}$ has distance 2 from $v_{i}, v_{i-1}$, and $v_{i+2}$. We pick coordinates and evaluate the length $\left|v-v^{\prime}\right|$. We find that $\left|v-v^{\prime}\right| \approx 1.746$, contrary to the hypothesis that the centers of the spheres of our packing are separated by distances of at least 2 . Thus, the hypothesis that $v$ has distance 2 from three other vertices is incorrect.
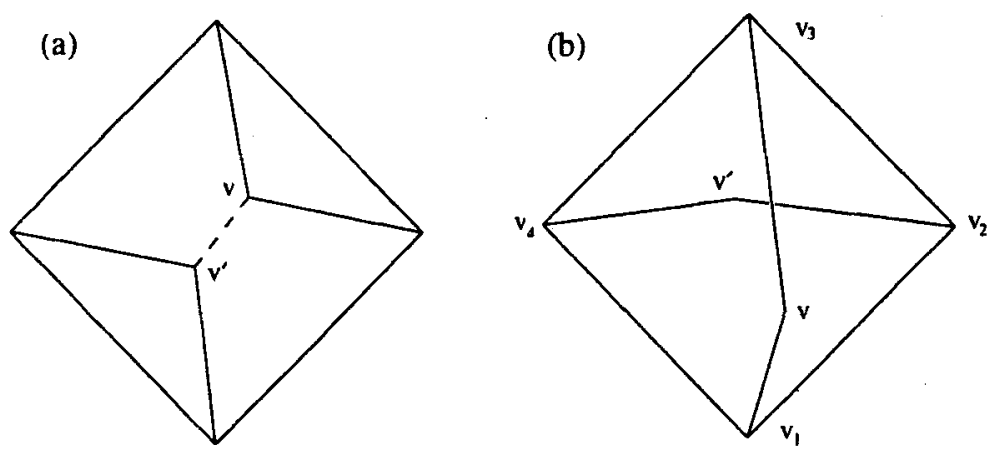

Diagram 4.4

We are left with one of the configurations of Diagram 4.4. An edge is drawn in the diagram, when the distance between the two endpoints has the smallest possible value (that is, 2.51 for the four edges of the quadrilateral, and 2 for the remaining edges). Deform the figure of case (a) along the one remaining degree of freedom until $\left|v-v^{\prime}\right|=2$. In case (a), referring to the notation established by Diagram 4.5, we have a quadrilateral on the unit sphere of edges $t_{1}=2 \arcsin (2.51 / 4) \approx 1.357$ radians, $t_{2}=\arccos (2.51 / 4)$ radians, and $t_{3}=2 \arcsin (1 / 2.51)$ radians. The form of this quadrilateral is determined by the angle $\alpha$, and it is clear that the angle $\beta(\alpha)$ is decreasing in $\alpha$. We have

$$
\theta=\operatorname{dih}_{\max }=\operatorname{dih}(S(2.51,2,2,2.51,2,2)) \approx 1.874444 .
$$

The figure exists if and only if there exists $\alpha$ such that $\beta(\alpha)+\beta(2 \pi-\theta-\alpha)=2 \pi-\theta$. 
By symmetry, we may assume that $\alpha \leq \pi-\theta / 2 \approx 2.20437$. The condition $\left|v-v_{1}\right| \geq 2$ implies that

$$
\alpha \geq \arccos \left(\frac{\cos t_{2}-\cos t_{2} \cos t_{3}}{\sin t_{2} \sin t_{3}}\right)>1.21
$$

However, by monotonicity,

$$
\beta(\alpha)+\beta(2 \pi-\theta-\alpha)<\beta\left(\alpha_{i}\right)+\beta\left(2 \pi-\theta-\alpha_{i}-0.1\right)<2 \pi-\theta,
$$

for $\alpha_{i} \leq \alpha \leq 0.1+\alpha_{i}$, with $\alpha_{i}=1.21+0.1 i$, and $i=0,1, \ldots, 9$, as a direct calculation of the constants $\beta\left(\alpha_{i}\right)+\beta\left(2 \pi-\theta-\alpha_{i}-0.1\right)$ will reveal. (The largest constant, which is about $2 \pi-\theta-0.113$, occurs for $i=0$.) Hence, the figure of Diagram 4.4(a) does not exist.

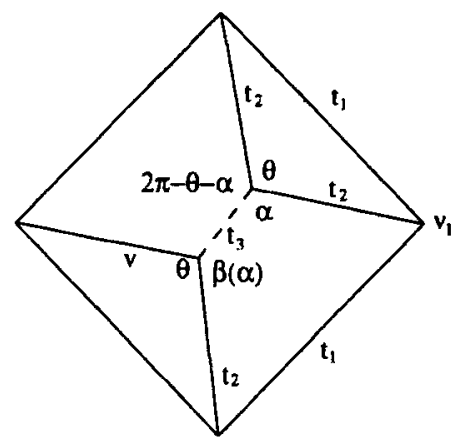

Diagram 4.5

To rule out Diagram 4.4(b), we reflect $v$, if necessary, to its image through the plane $P$ through $\left(0, v_{1}, v_{3}\right)$, so that $P$ separates $v$ and $v^{\prime}$. The vertex $v$ can then be pivoted away from $v^{\prime}$ along the axis through $v_{1}$ and $v_{3}$. This decreases $|v|$, but we may rescale so that $|v|=2.51$. Eventually $\left|v-v_{2}\right|=2$ or $\left|v-v_{4}\right|=2$. This is the previously considered case in which $v$ has distance 2 from three of the vertices $v_{i}$. This completes the proof that the original arrangement of two enclosed vertices does not exist.

\section{Restrictions}

If a Delaunay star $D^{*}$ is composed entirely of tetrahedra, then we obtain a triangulation of the unit sphere. As explained in Section 2, we wish to prove that no matter what the triangulation is, we always obtain a score less than $8 p t$. In this section we make a long list of properties that a configuration must have if it is to have a score of $8 \mathrm{pt}$ or more. The next section and the Appendix show that only one triangulation satisfies all of these properties. Additional arguments will show that this triangulation scores less than $8 p t$. This will complete the proof of Theorem 1 .

In this section the term vertices refers to the vertices of the triangulation of the unit sphere. The edges of the triangulation give a planar graph. We adopt the standard terminology of graph theory to describe the triangulation. We speak of the degree of a 
vertex, adjacent vertices, and so forth. The $n$ triangles around a vertex are referred to as an $n$-gon. We also refer to the corresponding $n$ tetrahedra that give the triangles of the polygon. We say that a triangulation contains a pattern $\left(a_{1}, \ldots, a_{n}\right)$, for $a_{i} \in \mathbb{N}$, if there are distinct vertices $v_{i}$ of degrees $a_{i}$ that are pairwise nonadjacent, for $i=1, \ldots, n$. Let $N$ be the number of vertices in the triangulation, and let $N_{i}$ be the number of vertices of degree $i$. We have $N=\sum N_{i}$.

In this section we start to use our various inequalities from Section 9 related to the score. Since the score $\sigma(S)$ may be either $\Gamma(S)$ or vor $(S)$ depending on the circumradius of $S$, there are two cases to consider for every inequality. In general, the inequalities for $\Gamma(S)$ are more difficult to establish. In the following sections we only cite the inequalities pertaining to $\Gamma(S)$. Section 9 shows how all the same inequalities hold for $\operatorname{vor}(S)$.

Proposition 5.1. Consider a Delaunay star $D^{*}$ that is composed entirely of quasiregular tetrahedra. Suppose that $\sigma\left(D^{*}\right) \geq 8$ pt. Then the following restrictions hold on the triangulation of the unit sphere given by the standard decomposition:

1. $13 \leq N \leq 15$.

2. $N=N_{4}+N_{5}+N_{6}$.

3. A region bounded by three edges is either a single triangle or the complement of a single triangle.

4. Two degree 4 vertices cannot be adjacent.

5. $\mathrm{N}_{4} \leq 2$.

6. Patterns $(6,6,6)$ and $(6,6,4)$ do not exist.

7. The pattern $(6,6)$ or $(6,4,4)$ implies that $N \geq 14$.

8. If there are two adjacent degree 6 vertices, and a third degree 6 vertex adjacent to neither of the first two, then $N=15$ and all other vertices are adjacent to at least one of these three.

9. The triangulation is made of geodesic arcs on the sphere whose radian lengths are between 0.8 and 1.36 .

Lemma 5.2. Consider a vertex of degree $n$, for some $4 \leq n \leq 7$. Let $S_{1}, \ldots, S_{n}$ be the tetrahedra that give the $n$ triangles. Then $\sum_{i=1}^{n} \sigma\left(S_{i}\right)$ is less than $z_{n}$, where $z_{4}=0.33 p t$, $z_{5}=4.52 p t, z_{6}=-1.52 p t$, and $z_{7}=-8.9$ pt. Suppose that $n \geq 6$. Let $S_{1}, \ldots, S_{4}$ be any four of the $n$ tetrahedra around the vertex. Then $\sum_{i=1}^{4} \sigma\left(S_{i}\right)<1.5 p t$.

Proof. When $n=4$, this is Lemma 9.5. When $n=5$, this is Lemma 9.6. When $n=6$ or $n=7$, we have, by Calculation 9.4 ,

$$
\begin{aligned}
\sum_{i=1}^{n} \sigma\left(S_{i}\right) & <\sum_{i=1}^{n}\left(0.378979 \operatorname{dih}\left(S_{i}\right)-0.410894\right) \\
& =2 \pi(0.378979)-0.410894 n .
\end{aligned}
$$

The right-hand side evaluates to about $-1.520014 \mathrm{pt}$ and $-8.940403 \mathrm{pt}$, respectively, when $n=6$ and $n=7$.

Assume that $n \geq 6$, and select any four $S_{1}, \ldots, S_{4}$ of the $n$ tetrahedra around the vertex. Let $S_{5}$ and $S_{6}$ be two other tetrahedra around the vertex. The dibedral angles of 
$S_{5}$ and $S_{6}$ are at least dih ${ }_{\min }$, by Calculation 9.3. Each of the four triangles (associated with $\left.S_{1}, \ldots, S_{4}\right)$ must then, on average, have an angle at most $\left(2 \pi-2 \operatorname{dih}_{\min }\right) / 4$ at $v$. By Calculation 9.4,

$$
\begin{aligned}
\sum_{i=1}^{4} \sigma\left(S_{i}\right) & <\sum_{i=1}^{4}\left(0.378979 \operatorname{dih}\left(S_{i}\right)-0.410894\right) \\
& \leq\left(2 \pi-2 \operatorname{dih}_{\min }\right) 0.378979+4(-0.410894)<1.5 p t .
\end{aligned}
$$

Proof of Proposition 5.1. Let $t=2(N-2)$ be the number of triangles, an even number. By Euler's theorem on polyhedra,

$$
3 N_{3}+2 N_{4}+N_{5}+0 N_{6}-N_{7} \cdots=12 .
$$

Let $S_{1}, \ldots, S_{t}$ denote the tetrahedra of $D^{*}$. Let $\sigma_{i}=\sigma\left(S_{i}\right)$ be the corresponding score. Let sol $_{i}$ denote the solid angle cut out by $S_{i}$ at the origin. We have $\sum \operatorname{sol}_{i}=4 \pi$. Often, without warning, we rearrange the indices $i$ so that the tetrahedra that give the triangles around a given vertex are numbered consecutively. When a vertex $v$ of the triangulation has been fixed, we let $\alpha_{i}$ denote the angles of the triangles at $v$, so that $\sum \alpha_{i}=2 \pi$. The angle $\alpha_{i}$ of a triangle is equal to the corresponding dihedral angle of the simplex $S_{i}$. We abbreviate certain sums over $n$ elements to $\sum_{(n)}$, when the context makes the indexing set clear. Throughout the argument, we use Calculation 9.1, which asserts that $\sigma_{i} \leq 1 \mathrm{pt}$, for all $i$. The proofs show that if a triangulation fails to have any of properties $1-9$, then the total score must be less than $8 \mathrm{pt}$.

(Proof of 5.1.1.) Assume that $t \geq 28$. By Calculation 9.9,

$$
\begin{aligned}
\sum_{(t)} \sigma_{i} & <\sum_{(t)}\left(0.446634 \mathrm{sol}_{i}-0.190249\right) \leq 4 \pi(0.446634)+t(-0.190249) \\
& \leq 4 \pi(0.446634)+28(-0.190249) \leq 8 p t .
\end{aligned}
$$

Suppose that $t \leq 18$. By Calculation 9.8 ,

$$
\begin{aligned}
\sum_{(t)} \sigma_{i} & <\sum_{(t)}\left(-0.37642101 \text { sol }_{i}+0.287389\right)=4 \pi(-0.37642101)+t(0.287389) \\
& \leq 4 \pi(-0.37642101)+18(0.287389) \leq 8 p t .
\end{aligned}
$$

This proves that $12 \leq N \leq 15$. The case $N=12$ is excluded after 5.1.2.

(Proof of 5.1.2.) By Lemma 8.3.2 and Calculation 9.3, each angle $\alpha$ of each triangle satisfies $\operatorname{dih}_{\min } \leq \alpha \leq \mathrm{dih}_{\max }$. In particular, $\alpha>\pi / 4$, so that each vertex has degree less than 8 , and $\alpha<2 \pi / 3$, so that each vertex has degree greater than 3 .

Assume for a contradiction that there is a vertex of degree 7 . Consider the seven tetrahedra around the given vertex. By Lemma 5.2, the tetrahedra satisfy

$$
\sum_{(7)} \sigma_{i}<-8.9 p t
$$

Suppose $t \leq 24$. For each vertex $v$, set $\zeta_{v}=\sum_{v} \sigma_{i}$, the sum running over the tetrahedra around $v$. Clearly, $\sum_{(t)} \sigma_{i}=\left(\sum_{v} \zeta_{v}\right) / 3$. Pick a vertex $v$ that is not a vertex of any of the 
seven triangles of the heptagon. By Lemma 5.2, we see that $\zeta_{v}<0.33 p t$ if $v$ has degree $4, \zeta_{v}<4.52 p t$ if $v$ has degree $5, \zeta_{v}<-1.52 p t$ if $v$ has degree 6 , and $\zeta_{v}<-8.9 p t$ if $v$ has degree 7. In particular, if $v$ has degree $n$, then $\zeta_{v}$ falls short of $n$ points by at least $0.48 \mathrm{pt}$. Thus,

$$
\sum_{(t)} \sigma_{i}<\sum_{(7)} \sigma_{i}+\sum_{(t-7)} p t-0.48 p t \leq(-8.9+(24-7)-0.48) p t<8 p t .
$$

Finally, we assume that $t=26$ and $N=15$. If $N_{6}=0$ and $N_{7}=1$, then Euler's theorem gives the incompatible conditions $2 N_{4}+N_{5}-1=12$ and $N_{4}+N_{5}+1=15$. Thus, $N_{6}>0$ or $N_{7}>1$. This gives a second $k$-gon $(k=6$ or 7$)$ around a vertex $v$. This second polygon shares at most two triangles with the original heptagon. This leaves at least four triangles of a second polygon exterior to the first. Thus, by Lemma 5.2,

$$
\sum_{(26)} \sigma_{i} \leq \sum_{(7)} \sigma_{i}+\sum_{(4)} \sigma_{i}+\sum_{(t-11)} p t<-8.9 p t+1.5 p t+(26-11) p t<8 p t .
$$

(Proof of 5.1.1, continued) Assume $N=12$. We define three classes of quasi-regular tetrahedra. In the first class all the edges have lengths between 2 and 2.1. In the second class the fourth, fifth, and sixth edges have lengths greater than 2.1 . The third class is everything else.

Set $\varepsilon=0.001, a=-0.419351, b=0.2856354$. The following are established by Calculations 9.10-9.12:

$$
\begin{array}{ll}
\sigma(S) \leq a \operatorname{sol}(S)+b+\varepsilon & \text { for } S \text { in the first class, } \\
\sigma(S) \leq a \operatorname{sol}(S)+b & \text { for } S \text { in the second class, } \\
\sigma(S) \leq a \operatorname{sol}(S)+b-5 \varepsilon & \text { for } S \text { in the third class. }
\end{array}
$$

Consider a vertex $v$ of degree $n=4,5$, or 6 and the surrounding tetrahedra $S_{1}, \ldots, S_{n}$. We claim that

$$
\sum_{i=1}^{n} \sigma\left(S_{i}\right) \leq \sum_{i=1}^{n}\left(a \operatorname{sol}\left(S_{i}\right)+b\right) .
$$

This follows directly from the stated inequalities if none of these tetrahedra are in the first class. It is also obvious if at least one of these tetrahedra is in the third class, because then the inequality is violated by at most $-5 \varepsilon+(n-1) \varepsilon \leq 0$. So assume that all of the tetrahedra are in the first two classes with at least one in the first class. By the restrictions on the lengths of the edges, a tetrahedron in the first class cannot be adjacent to one in the second class. We conclude that the tetrahedra are all in the first class.

By Lemma 5.2, $z_{n} \leq n(0.904) p t$. If $\sum_{(n)} \operatorname{sol}\left(S_{i}\right) \leq n(0.56176)$, then

$$
\sum_{(n)} \sigma\left(S_{i}\right) \leq n(0.904) p t \leq \sum_{(n)}(a(0.56176)+b) \leq \sum_{(n)}\left(a \operatorname{sol}\left(S_{i}\right)+b\right) .
$$

So we may assume that $\sum_{(n)} \operatorname{sol}\left(S_{i}\right) \geq n(0.56176)$. By Calculation 9.13 ,

$$
\sum_{(n)} \sigma\left(S_{i}\right) \leq \sum_{(n)}\left(-0.65557 \operatorname{sol}\left(S_{i}\right)+0.418\right)
$$




$$
\begin{aligned}
& \leq \sum_{(n)}\left(a \operatorname{sol}\left(S_{i}\right)+b\right)+\sum_{(n)}(0.132365-0.236219(0.56176)) \\
& <\sum_{(n)}\left(a \operatorname{sol}\left(S_{i}\right)+b\right)
\end{aligned}
$$

This establishes inequality (5.1.1.1). By averaging over every vertex, we see that the average of the scores $\sigma(S)$ is less than the average of $a \operatorname{sol}(S)+b$. So

$$
\sigma\left(D^{*}\right)=\sum_{(t)} \sigma(S) \leq \sum_{(t)}(a \operatorname{sol}(S)+b)=4 \pi a+20 b \approx 7.99998 p t<8 p t .
$$

(Proof of 5.1.3.) This is Lemma 3.7. (If we had not introduced quasi-regular tetrahedra, then this result would no longer hold.)

(Proof of 5.1.4.) If two degree 4 vertices are adjacent, we then have the arrangement of Diagram 5.3. This is a quadrilateral enclosing two vertices, contrary to Proposition 4.2.

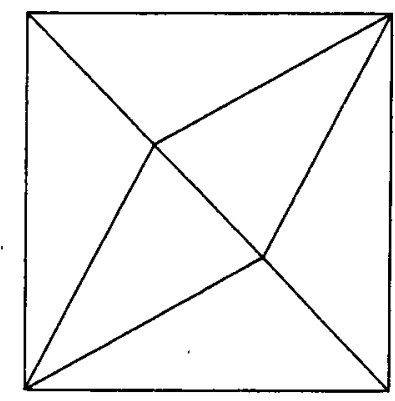

Diagram 5.3

(Proof of 5.1.5.) As in the proof of 5.1.2, for each vertex $v$, set $\zeta_{v}=\sum_{v} \sigma_{i}$, the sum running over the tetrahedra around the vertex $v$. We use the estimates of $\zeta_{v}$ that appear in Lemma 5.2 .

We have found that $N_{i}=0$, if $i \neq 4,5,6$. By Euler's theorem, $N_{5}=12-2 N_{4}$ and $N_{6}=N_{4}+N-12$. Assume that $N \geq 13$ and that $N_{4} \geq 3$. Then

$$
\begin{aligned}
\sum_{(t)} \sigma_{i} & =\frac{1}{3} \sum_{(N)} \zeta_{v}<\frac{1}{3}\left(0.33 N_{4}+4.52\left(12-2 N_{4}\right)-1.52\left(N_{4}+N-12\right)\right) p t \\
& \leq \frac{1}{3}(0.33(3)+4.52(12-6)-1.52(3+13-12)) p t<8 p t
\end{aligned}
$$

(Proof of 5.1.6.) Suppose that we have the pattern $(6,6,6)$ Then reordering indices according to the polygons in the pattern, we have, by the estimates of Lemma 5.2,

$$
\begin{aligned}
\sum_{(t)} \sigma_{i} & \leq \sum_{(6)} \sigma_{i}+\sum_{(6)} \sigma_{i}+\sum_{(6)} \sigma_{i}+\sum_{(t-18)} \sigma_{i} \\
& <-1.52 p t-1.52 p t-1.52 p t+(26-18) p t<3.5 p t .
\end{aligned}
$$

Similarly, if we have the pattern $(6,6,4)$, then we find

$$
\sum_{(t)} \sigma_{i} \leq-1.52 p t-1.52 p t+0.33 p t+(26-16) p t<8 p t .
$$


(Proof of 5.1.7.) We use the same method as in the proof of 5.1.6. If we have the pattern $(6,6)$, and if $t \leq 22$, then

$$
\sum_{(t)} \sigma_{i} \leq \sum_{(6)} \sigma_{i}+\sum_{(6)} \sigma_{i}+\sum_{(t-12)} \sigma_{i} \leq-1.52 p t-1.52 p t+(22-12) p t<8 p t .
$$

Similarly, if we have the pattern $(6,4,4)$, then $\sum_{(t)} \sigma_{i}$ is less than $-1.52 p t+0.33 p t+$ $0.33 p t+8 p t<8 p t$.

(Proof of 5.1.8.) Let the two adjacent degree 6 vertices be $v_{1}$ and $v_{2}$. Let the third be $v_{3}$. The six triangles in the hexagon around $v_{3}$ give less than $-1.52 \mathrm{pt}$. The ten triangles in the hexagons around $v_{1}$ and $v_{2}$ give at most

$$
\sum_{(4)} \sigma_{i} \leq \sum_{(6)} \sigma_{i}<1.5 p t-1.52 p t<0 p t,
$$

by the argument described in the case $t=26$ of 5.1 .2 (see Lemma 5.2).

Suppose that $t \leq 24$. There remain at most $24-16=8$ triangles, and they give a combined score of at most $8 \mathrm{pt}$. The total score is then less than $(-1.52+8) p t$, as desired.

Now assume that $t=26$. Suppose there is a vertex $v$ that is not adjacent to any of $v_{1}$, $v_{2}$, or $v_{3}$. As in the proof of 5.1.8, the ten triangles in the two overlapping hexagons give less than $0 p t$. The other hexagon gives less than $-1.52 \mathrm{pt}$. By Lemma 5.2, the $n$ triangles around $v$ fall short of $n$ points by at least $(5-4.52) p t=0.48 p t$. Each of the remaining triangles gives at most $1 \mathrm{pt}$. The score is then less than $(0-1.52+(26-16)-0.48) p t=$ $8 p t$, as desired.

(Proof of 5.1.9.) This follows directly from the construction of the triangulation and the close-neighbor restrictions on the lengths of the edges of a quasi-regular tetrahedron. The lengths are between $0.8<2 \arcsin (1 / 2.51)$ and $2 \arcsin (2.51 / 4)<1.36$. This completes the proof of the proposition.

\section{Combinatorics}

Theorem 6.1. Suppose that a triangulation satisfies Proposition 4.2 and properties 1-9 of Proposition 5.1. Then it must be the triangulation of Diagram 6.2 with 14 vertices and 24 triangles.

Proof. Fix a polygon centered at a vertex $u_{0}$, such as the hexagon in Diagram 6.3. The six vertices $v_{1}, \ldots, v_{6}$ of the hexagon are distinct, for otherwise two distinct geodesic
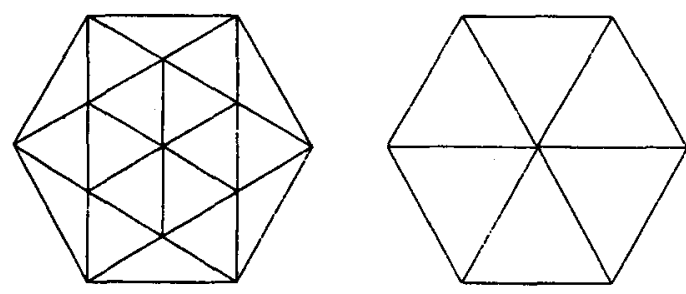

Diagram 6.2 


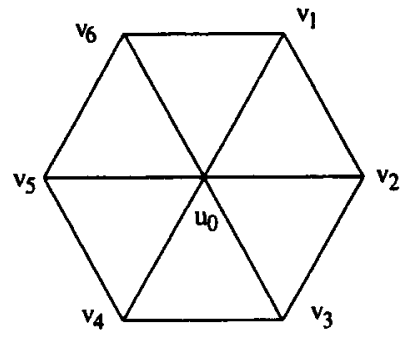

Diagram 6.3

arcs on the sphere would run between $u_{0}$ and $v_{i}$ for some $i$. This is impossible, because $u_{0}$ and $v_{i}$ are not antipodal by Property 5.1.9. Similarly, the vertices of every other polygon of the triangulation are distinct.

We then extend the polygon to a second layer of triangles. Each of the vertices $v_{i}$ has degree 4,5 , or 6 , and two degree 4 vertices cannot be adjacent. The new vertices are denoted $w_{1}, \ldots, w_{k}$. One example is shown in Diagram 6.4.

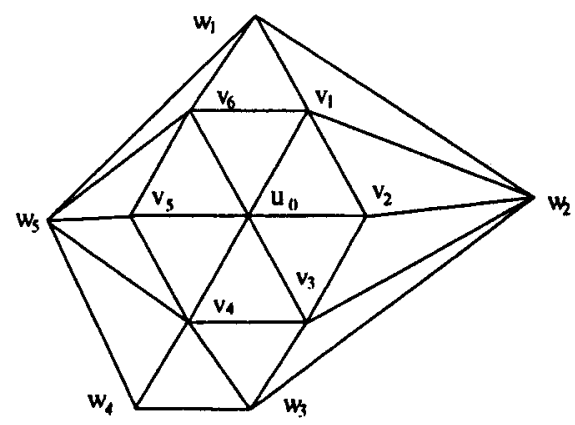

Díagram 6.4

There can be no identification of a vertex $v_{i}$ with a vertex $w_{j}$, for otherwise there is a triangle (say with vertices $w_{j}, v_{k}, u_{0}, v_{i}=w_{j}$ ) that is subtriangulated, contrary to Property 5.1.3. Similarly, there is no identification of two vertices $w_{i}$ and $w_{j}$, for otherwise it can be checked that there is a quadrilateral (with vertices $w_{i}, v_{k}, u_{0}, v_{\ell}, w_{j}=w_{i}$ ) that encloses more than one vertex, which is impossible by Proposition 4.2. A purely combinatorial problem remains. It is solved in the Appendix.

Proposition 6.5. The triangulation of Theorem 6.1 scores less than 8 pt.

Proof. Our initial bound on the score comes by viewing the triangulation as made up of two hexagons and twelve additional triangles. By Lemma 5.2 and Calculation 9.1,

$$
\sum_{(24)} \sigma_{i} \leq \sum_{(6)} \sigma_{i}+\sum_{(6)} \sigma_{i}+\sum_{(12)} p t<-1.52 p t-1.52 p t+12 p t=8.96 p t
$$

A refinement is required in order to lower the upper bound to $8 \mathrm{pt}$. 
If a vertex $v$ has height $|v| \geq 2.2$, then, by Calculation 9.2 , we find $\sigma_{i}<0.5 p t$ for the tetrahedra at $v$. Thus, in inequality (6.6), if the vertex $v$ of some pentagon has height $|v| \geq 2.2$, then the term $\sum_{(12)} p t$ may be replaced by $\sum_{(9)} p t+\sum_{(3)} 0.5 p t$ (there are three triangles in the pentagon that do not belong to the hexagon), and the upper bound on the score falls to $7.46 \mathrm{pt}$.

If a vertex of degree 6 has height $|v| \geq 2.05$, then we claim that $\sum_{(6)} \sigma_{i}<-3.04 p t$. In fact, by Calculation 9.7, the hexagon gives

$$
\begin{aligned}
\sum_{(6)} \sigma_{i} & <\sum_{(6)}\left(0.389195 \operatorname{dih}\left(S_{i}\right)-0.435643\right) \\
& =2 \pi(0.389195)-6(0.435643)<-3.04 p t .
\end{aligned}
$$

Estimate (6.6) is improved to

$$
\sum_{(24)} \sigma_{i}<-3.04 p t-1.52 p t+12 p t=7.44 p t
$$

If the twelve tetrahedra have combined solid angle less than 6.48 , then, by Calculation 9.9 ,

$$
\begin{aligned}
\sum_{(12)} \sigma_{i} & <\sum_{(12)}\left(0.446634 \operatorname{sol}\left(S_{i}\right)-0.190249\right) \\
& \leq 6.48(0.446634)+12(-0.190249)<11.039 p t
\end{aligned}
$$

Then estimate (6.6) is improved to the bound $-1.52 p t-1.52 p t+11.039 p t=7.999 p t$.

Now assume, on the other hand, that the combined solid angle of the two hexagons is at most $4 \pi-6.48$. Set $K=(4 \pi-6.48) / 12$ and define $\sigma^{\prime}(S):=\sigma(S)+(K-\operatorname{sol}(S)) / 3$. The solid angle of one of the two hexagons is at most $6 K$. For that hexagon, we have

$$
\sum_{(6)} \sigma^{\prime}\left(S_{i}\right)=\sum_{(6)} \sigma_{i}+\frac{6 K-\sum_{(6)} \operatorname{sol}\left(S_{i}\right)}{3} \geq \sum_{(6)} \sigma_{i} .
$$

By our previous estimates, we now assume without loss of generality that the heights $|v|$ of the vertices of triangles in the hexagon are at most $2.05,2.2$, and 2.2 , the bound of 2.05 occurring at the center of the hexagon. By Calculation 9.15 ,

$$
\begin{aligned}
\sum_{(6)} \sigma^{\prime}\left(S_{i}\right) & \leq \sum_{(6)} 0.564978 \operatorname{dih}_{i}-0.614725 \\
& =2 \pi(0.564978)+6(-0.614725)<-2.5 p t .
\end{aligned}
$$

Estimate (6.6) becomes

$$
\sum_{(24)} \sigma_{i} \leq \sum_{(6)} \sigma^{\prime}\left(S_{i}\right)+\sum_{(6)} \sigma_{i}+\sum_{(12)} \sigma_{i}<(-2.5-1.52+12) p t=7.98 p t .
$$

This completes the proof of the proposition. 


\section{The Method of Subdivision}

The rest of this paper is devoted to the verification of the inequalities that have been used in Sections 5 and 6. In this section we describe the method used to obtain several of our bounds. We call it the method of subdivision. Let $p(x)=\sum_{I} c_{I} x^{I}$ be a polynomial with real coefficients $c_{I}$, where $I=\left(i_{1}, \ldots, i_{n}\right), x=\left(x_{1}, \ldots, x_{n}\right) \in \mathbb{R}^{n}$, and $x^{I}=x_{1}^{i_{1}} \ldots x_{n}^{i_{n}}$. It is clear that if $C$ is the product of intervals $\left[a_{1}, b_{1}\right] \times \cdots \times\left[a_{n}, b_{n}\right] \subset \mathbb{R}^{n}$ in the positive orthant $\left(a_{i}>0\right.$, for $\left.i=1, \ldots, n\right)$, then

$$
\forall x \in C, \quad p_{\min }(C) \leq p(x) \leq p_{\max }(C),
$$

where

$$
p_{\min }(C)=\sum_{c_{l}>0} c_{I} a^{I}+\sum_{c_{l}<0} c_{l} b^{I} \quad \text { and } \quad p_{\max }(C)=\sum_{c_{l}>0} c_{l} b^{I}+\sum_{c_{l}<0} c_{l} a^{I} .
$$

Another bound comes from the Taylor polynomial $p(x)=\sum d_{I}(x-a)^{I}$ at $a$ :

$$
d_{0}+\sum_{d_{I}<0, I \neq 0} d_{I}(b-a)^{I} \leq p(x) \leq d_{0}+\sum_{d_{I}>0, I \neq 0} d_{I}(b-a)^{I} .
$$

If $r(x)=p(x) / q(x)$ is a rational function, and if $q_{\min }(C)>0$, then

$$
\forall x \in C, \quad \frac{p_{\min }(C)}{q_{1}(C, p)} \leq r(x) \leq \frac{p_{\max }(C)}{q_{2}(C, p)},
$$

where $q_{1}(C, p)$ (resp. $q_{2}(C, p)$ ) is defined as $q_{\max }(C)$ whenever $p_{\min }(C) \geq 0$ (resp. $\left.p_{\max }(C)<0\right)$ and as $q_{\min }(C)$ otherwise.

We define a cell to be a product of intervals in the positive orthant of $\mathbb{R}^{n}$. By covering a region with a sufficiently fine collection of cells, various inequalities of rational functions are easily established. To prove an inequality of rational functions with positive denominators (say $r_{1}(x)<r_{2}(x)$, for all $x \in C$ ), we cover $C$ with a finite number of cells and compare the upper bound of $r_{1}(x)$ with the lower bound of $r_{2}(x)$ on each cell. If it turns out that some of the cells give too coarse a bound, then we subdivide each of the delinquent cells into a number of smaller cells and repeat the process. If at some stage we succeed in covering the original region $C$ with cells on which the upper bound of $r_{1}(x)$ is less than the lower bound of $r_{2}(x)$, the inequality is established.

A refinement of this approach applies the method to the partial derivatives. If, for instance, we establish by the method of subdivision that, for some $i$,

$$
\frac{\partial p}{\partial x_{i}}(x) \geq 0, \quad \forall x \in C,
$$

then we may compute an upper bound of $p$ by applying the method of subdivision to the polynomial obtained from $p$ by the specialization $x_{i}=b_{i}$, where $b_{i}$ is the upper bound of $x_{i}$ on $C$. Thus, we obtain an upper bound on a polynomial by fixing all the variables that are known to have partial derivatives of fixed sign and then applying the method subdivision to the resulting polynomial. Similar considerations apply to lower bounds and to rational functions with positive denominators. 
It is a fortunate circumstance that many of the polynomials we encounter in sphere packings are quadratic in each variable with negative leading coefficient $(u, \rho, \Delta, \chi$ in the next section). In this case the lower bound is attained at a corner of the cell. Of course, the maximum of a quadratic function with negative leading coefficient is also elementary: $-\alpha\left(x-x_{0}\right)^{2}+\beta \leq \beta$, if $\alpha \geq 0$.

\section{Explicit Formulas for Compression, Volume, and Angle}

Many of the formulas in this section are classical. They can typically be found in 19th century primers on solid geometry. The formula for solid angles, for example, is due to Euler and Lagrange. For anyone equipped with symbolic algebra software, the verifications are elementary, so we omit many of the details. All formulas in this section are valid for Delaunay simplices and for quasi-regular tetrahedra, unless otherwise noted.

\subsection{The Volume of a Simplex}

As in the previous section, a cell in $\mathbb{R}^{n}$ is a product of intervals in $\mathbb{R}^{n}$. We define a function $\Delta:[4,16]^{6} \subset \mathbb{R}^{6} \rightarrow \mathbb{R}$ by

$$
\begin{aligned}
\Delta\left(x_{1}, \ldots, x_{6}\right)= & x_{1} x_{4}\left(-x_{1}+x_{2}+x_{3}-x_{4}+x_{5}+x_{6}\right) \\
& +x_{2} x_{5}\left(x_{1}-x_{2}+x_{3}+x_{4}-x_{5}+x_{6}\right) \\
& +x_{3} x_{6}\left(x_{1}+x_{2}-x_{3}+x_{4}+x_{5}-x_{6}\right) \\
& -x_{2} x_{3} x_{4}-x_{1} x_{3} x_{5}-x_{1} x_{2} x_{6}-x_{4} x_{5} x_{6} .
\end{aligned}
$$

We set $y_{i}=\sqrt{x_{i}}$, for $i=1, \ldots, 6$. This relationship between $x_{i}$ and $y_{i}$ remains in force to the end of the paper. Index the edges of a simplex as in Diagram 8.1.2.

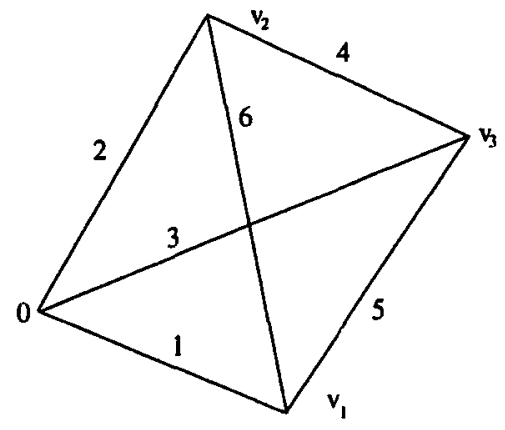

Diagram 8.1.2

We also define $u:[4,16]^{3} \rightarrow \mathbb{R}$ by

$$
\begin{aligned}
u\left(x_{1}, x_{2}, x_{6}\right)= & \left(y_{1}+y_{2}+y_{6}\right)\left(y_{1}+y_{2}-y_{6}\right)\left(y_{1}-y_{2}+y_{6}\right) \\
& \times\left(-y_{1}+y_{2}+y_{6}\right) \\
= & -x_{1}^{2}-x_{2}^{2}-x_{6}^{2}+2 x_{1} x_{6}+2 x_{1} x_{2}+2 x_{2} x_{6} .
\end{aligned}
$$


Lemma 8.1.4. There exists a simplex of positive volume with edges of length $y_{1}, \ldots, y_{6}$ if and only if $\Delta\left(x_{1}, \ldots, x_{6}\right)>0$. If these conditions hold, then the simplex has volume $\Delta\left(x_{1}, \ldots, x_{6}\right)^{1 / 2} / 12$.

Proof. The function $u=u\left(x_{1}, x_{2}, x_{6}\right)$ is quadratic in each variable, with negative leading coefficient, so the minimum of $u$, which is 0 , is attained at a vertex of the cube $[4,16]^{3}$. At the vertices where the minimum is attained, $\Delta\left(x_{1}, \ldots, x_{6}\right) \leq 0$.

Assume $\Delta>0$. Then $u>0$, and a simplex exists with vertices $0, X=\left(y_{1}, 0,0\right)$, $Y=\frac{1}{2}\left(*, u^{1 / 2} / y_{1}, 0\right), Z=\left(*, *,(\Delta / u)^{1 / 2}\right)$. Conversely, if the simplex exists, then it must be of the given form, up to an orthogonal transformation, so $u>0$ (otherwise $O$, $X$, and $Y$ are colinear) and $\Delta>0$. The volume is $|\operatorname{det}(X, Y, Z)| / 6=\Delta^{1 / 2} / 12$.

Let $C$ be a cell contained in $[4,16]^{6}$. The minimum of $\Delta$ on $C$ is attained at a vertex of $C$ : this is clear because $\Delta$ is quadratic in each variable with negative leading coefficient. To obtain an upper bound on $\Delta$ on a cell, we may use the method of Section 7 . The restriction of $\Delta$ to the zero set of $\partial \Delta / \partial x_{1}$ is

$$
\frac{u\left(x_{4}, x_{5}, x_{6}\right) \cdot u\left(x_{2}, x_{3}, x_{4}\right)}{4 x_{4}} .
$$

This is an upper bound on $\Delta\left(x_{1}, \ldots, x_{6}\right)$.

\subsection{The Circumradius of a Simplex}

The circumradius of a face with edges $y_{4}, y_{5}$, and $y_{6}$ is

$$
\eta\left(y_{4}, y_{5}, y_{6}\right)=\frac{y_{4} y_{5} y_{6}}{u\left(y_{4}^{2}, y_{5}^{2}, y_{6}^{2}\right)^{1 / 2}} \text {. }
$$

Define $\rho:[4,16]^{6} \rightarrow \mathbb{R}$ by

$$
\rho\left(x_{1}, \ldots, x_{6}\right)=-x_{1}^{2} x_{4}^{2}-x_{2}^{2} x_{5}^{2}-x_{3}^{2} x_{6}^{2}+2 x_{1} x_{2} x_{4} x_{5}+2 x_{1} x_{3} x_{4} x_{6}+2 x_{2} x_{3} x_{5} x_{6} .
$$

Lemma 8.2.2. Suppose $\Delta>0$; then $\rho>0$.

Proof. If $\Delta>0$, then the simplex of Lemma 8.1.4 exists. Let the coordinates of the circumcenter of the simplex be $(x, y, z)$. Direct calculation shows that $0<x^{2}+y^{2}+z^{2}=$ $\rho /(4 \Delta)$.

Corollary 8.2.3. Let $S$ be a Delaunay simplex of positive volume with edges of lengths $y_{i}=\sqrt{x_{i}}$. The circumradius is $\frac{1}{2}(\rho / \Delta)^{1 / 2}$.

Set

$$
\begin{aligned}
\chi\left(x_{1}, x_{2}, x_{3}, x_{4}, x_{5}, x_{6}\right)= & x_{1} x_{4} x_{5}+x_{1} x_{6} x_{4}+x_{2} x_{6} x_{5}+x_{2} x_{4} x_{5}+x_{5} x_{3} x_{6} \\
& +x_{3} x_{4} x_{6}-2 x_{5} x_{6} x_{4}-x_{1} x_{4}^{2}-x_{2} x_{5}^{2}-x_{3} x_{6}^{2} .
\end{aligned}
$$


We have

$$
\frac{\rho}{4 \Delta}-\eta\left(y_{4}, y_{5}, y_{6}\right)^{2}=\frac{\chi\left(x_{1}, \ldots, x_{6}\right)^{2}}{4 u\left(x_{4}, x_{5}, x_{6}\right) \Delta} .
$$

The vanishing of $\chi$ is the condition for the circumcenter of the simplex to lie in the plane through the face $T$ bounded by the fourth, fifth, and sixth edges. $\chi$ is positive if the circumcenter of $S$ and the vertex of $S$ opposite $T$ lie on the same side of the plane through $T$ and negative if they lie on opposite sides of the plane. We say that $T$ has positive orientation when $\chi>0$.

8.2.4. The function $\rho$ is quadratic in each of the variables $x_{1}, \ldots, x_{6}$ with negative leading coefficient. Thus, the minimum of $\rho$ is attained at a vertex of a given cell $C$. The derivative is $\Delta^{2} \partial(\rho / \Delta) / \partial x_{1}=\chi\left(x_{5}, x_{6}, x_{1}, x_{2}, x_{3}, x_{4}\right) \chi\left(x_{1}, x_{2}, x_{3}, x_{4}, x_{5}, x_{6}\right)$. This leads to bounds on the circumradius by the method of Section 7 .

The circumradius of a quasi-regular tetrahedron is increasing in $x_{1}, \ldots, x_{6}$ if the orientation of each face is positive. When a face fails to have a positive orientation, it satisfies the constraints of Section 3 (for example, $y_{1}, y_{2}, y_{3} \in[2,2.15], y_{4}, y_{5}, y_{6} \in[2.3,2.51]$, $\eta\left(y_{4}, y_{5}, y_{6}\right) \geq \sqrt{2}$, if it is the face opposite the origin). This allows us to determine bounds on the circumradius for most of the quasi-regular tetrahedra we encounter in this paper by inspection. For example, in Section 9 we study the simplices constrained by $y_{i} \in[2,2.1]$. An upper bound on the circumradius is $\operatorname{rad}(S(2.1,2.1,2.1,2.1,2.1,2.1))$.

8.2.5. Proof of Lemma 3.4. In the notation of Section 3.4 let $T$ be the given face, and let $v_{0}$ be a vertex satisfying the conditions of the lemma. Let $S$ by the simplex at the origin $v_{0}=0$, whose first, second, and third edges abut at $v_{i}$, for $i=1,2,3$. Suppose for a contradiction that $\left|v_{0}-v_{3}\right|^{2}=x_{3} \geq 2.15^{2}$. In light of the results of this section, a contradiction follows if $\chi\left(x_{1}, \ldots, x_{6}\right)>0$, for $x_{i} \geq 4$ for $i=1,2, x_{3} \geq 2.15^{2}$, $2.3^{2} \leq x_{i} \leq 2.51^{2}$ for $i=4,5,6$. (The lower bound of 2.3 comes from Remarks 3.2 and 3.3: the circumradius of $T$ is at least $\sqrt{2}$ ). Since $T$ is acute,

$$
\frac{\partial \chi}{\partial x_{1}}=x_{4}\left(-x_{4}+x_{5}+x_{6}\right)>0 \text {. }
$$

Similarly, $\chi$ is increasing in $x_{2}$ and $x_{3}$. Thus, to minimize $\chi$, we take $x_{1}=x_{2}=4$, $x_{3}=2.15^{2}$. However, $\chi$ is quadratic in each variable $x_{4}, x_{5}$, and $x_{6}$ with negative leading coefficient, so the minimum occurs at a corner point of $\left[2.3^{2}, 2.51^{2}\right]^{3}$. We find $\chi \geq \chi\left(2^{2}, 2^{2}, 2.15^{2}, 2.51^{2}, 2.51^{2}, 2.51^{2}\right) \approx 0.885>0$.

For the set of Delaunay stars to be a compact topological space, simplices of zero volume must be included. Since the circumradius remains bounded, the degenerate Delaunay simplices of zero volume are planar quadrilaterals that possess a circumscribing circle.

\subsection{Dihedral Angles}

Let $\operatorname{dih}(S)$ be the dihedral angle of a simplex $S$ along the first edge. It is the (interior) angle formed by faces with edges $(2,1,6)$ and $(1,3,5)$. 
Lemma 8.3.1.

$$
\cos \operatorname{dih}(S)=\frac{\partial \Delta / \partial x_{4}}{\left(u\left(x_{1}, x_{2}, x_{6}\right) u\left(x_{1}, x_{3}, x_{5}\right)\right)^{1 / 2}},
$$

where $u$ is the function defined by (8.1.3).

The partial derivative of $\cos \operatorname{dih}(S)$ with respect to $x_{3}$ is

$$
\frac{2 x_{1}\left(\partial \Delta / \partial x_{2}\right)}{u\left(x_{1}, x_{2}, x_{6}\right)^{1 / 2} u\left(x_{1}, x_{3}, x_{5}\right)^{3 / 2}}
$$

so that the sign of the partial derivative is determined by the sign of $\partial \Delta / \partial x_{2}$. Similar considerations apply to the partial derivatives with respect to $x_{2}, x_{5}$, and $x_{6}$.

Lemma 8.3.2. Let $S$ be a quasi-regular tetrahedron. The the dihedral angle of $S$ along any edge is at most $\arccos (-29003 / 96999)=\operatorname{dih}_{\max } \approx 1.874444$.

Proof. Suppose that $\operatorname{dih}(S)>\pi / 2$, so that the numerator $n=\partial \Delta / \partial x_{4}$ in Lemma 8.3.1 is negative. To bound cos dih $(S)$ from below, we minimize $u\left(x_{1}, x_{2}, x_{6}\right), u\left(x_{1}, x_{3}, x_{5}\right)$, and the numerator $n$ over the variables $x_{2}, x_{3}, x_{5}$, and $x_{6}$.

We have $\partial_{i} u=x_{j}+x_{k}-x_{i}>0$ on the indicated domain, for $i=2,3,5$, and 6 , so that

$$
u\left(x_{1}, x_{2}, x_{6}\right) u\left(x_{1}, x_{3}, x_{5}\right) \geq u\left(x_{1}, 2^{2}, 2^{2}\right)^{2}=\left(-x_{1}^{2}+16 x_{1}\right)^{2} .
$$

Similarly, $\partial_{i} n=x_{1}+x_{j}-x_{k}>0$, for $i=2,3,5$, and 6 , so that

$$
n\left(x_{1}, \ldots, x_{6}\right)>n\left(x_{1}, 2^{2}, 2^{2}, x_{4}, 2^{2}, 2^{2}\right)=x_{1}\left(16-x_{1}-2 x_{4}\right) .
$$

Thus,

$$
\begin{aligned}
0 & \geq \cos \operatorname{dih}(S) \geq \frac{16-x_{1}-2 x_{4}}{16-x_{1}}=1-\frac{2 x_{4}}{16-x_{1}} \\
& \geq 1-\frac{2(2.51)^{2}}{16-2.51^{2}}=\frac{-29003}{96999}
\end{aligned}
$$

\subsection{The Solid Angles of a Delaunay Simplex}

Let $S$ be a Delaunay simplex with vertices $v_{0}, \ldots, v_{3}$. By the solid angle $\operatorname{sol}_{i}(S)$ of the simplex at the vertex $i$, we mean $3 \operatorname{vol}\left(S \cap B_{i}\right)$, where $B_{i}$ is a unit ball centered at $v_{i}$. For simplicity, suppose that $v_{0}$ is located at the origin, and that, in the notation of Diagram 8.1 .2 , the vertices $v_{1}, v_{2}, v_{3}$ are the edges of lengths $y_{1}, y_{2}$, and $y_{3}$. We write sol for solo. Set

$$
\begin{aligned}
a\left(y_{1}, y_{2}, \ldots, y_{6}\right)= & y_{1} y_{2} y_{3}+\frac{1}{2} y_{1}\left(y_{2}^{2}+y_{3}^{2}-y_{4}^{2}\right) \\
& +\frac{1}{2} y_{2}\left(y_{1}^{2}+y_{3}^{2}-y_{5}^{2}\right)+\frac{1}{2} y_{3}\left(y_{1}^{2}+y_{2}^{2}-y_{6}^{2}\right) .
\end{aligned}
$$


Lemma 8.4.2.

$$
\operatorname{sol}(S)=2 \operatorname{arccot}\left(\frac{2 a}{\Delta^{1 / 2}}\right)
$$

Proof. (See $[\mathrm{H} 2$, p. 64].) We use the branch of arccot taking values in $[0, \pi]$.

The function $a$ is increasing in $y_{1}, y_{2}$, and $y_{3}$ on $[2,4]$ and is decreasing in the variables $y_{4}, y_{5}$, and $y_{6}$ on the same interval.

We claim that $a>0$ for any Delaunay simplex. We prove this in the case $\Delta>0$, leaving the degenerate case $\Delta=0$ to the reader. The area of the face $T$ opposite the origin is at most $4 \sqrt{3}$ (since its edges are all at most 4 ). There exists a plane that is tangent to the unit sphere between this face and the unit sphere [H1, 2.1]. The area of the radial projection of $T$ to this plane is less than the area of a disk $D$ of radius 1.5 on the plane centered at the point of tangency. The solid angle (that is, the area of the radial projection of $T$ to the unit sphere) is less than the area of the radial projection of $D$ to the unit sphere). This area is $2 \pi(1-\cos (\arctan (1.5)))<\pi$. Lemma 8.4.2 now gives the result. This allows us to use Lemma 8.4.2 in the form $\operatorname{sol}(S)=2 \arctan \left(\Delta^{1 / 2} /(2 a)\right)$.

8.4.3. The solid angle is the area of a spherical triangle. Let $x, y$, and $z$ be the cosines of the radian lengths of the edges of the triangle. By the spherical law of cosines, the solid angle, expressed as a function of $x, y$, and $z$ is

$c(x, y, z)+c(y, z, x)+c(z, x, y)-\pi, \quad c(x, y, z):=\arccos \left(\frac{x-y z}{\sqrt{\left(1-y^{2}\right)\left(1-z^{2}\right)}}\right)$.

The partial derivative with respect to $x$ of this expression for the solid angle is $(-1-x+y+z) /((x+1) \sqrt{t})$, where $t=1-x^{2}-y^{2}-z^{2}+2 x y z$. The second derivative of this expression evaluated at $-1-x+y+z=0$ is $-2(1-y)(1-z) t^{-3 / 2} \leq 0$. So the unique critical point is always a local maximum. If neither edge at a vertex of a spherical triangle is constrained, then the triangle can be contracted or expanded by moving the vertex. If the lengths of the edges are constrained to lie in a product of intervals, then the minimum area occurs when two of the edges are as short as possible and the third is at one of the extremes. The maximum area is attained when two of the edges are as long as possible and the third is at one of the extremes or at a critical point: $x=y+z-1$ (or it symmetries, $y=x+z-1, z=x+y-1$ ).

\subsection{The Compression of a Delaunay Simplex}

The compression $\Gamma(S)$ of a Delaunay simplex $S$ is defined as

$$
\Gamma(S)=-\delta_{o c t} \operatorname{vol}(S)+\frac{\sum_{i=1}^{4} \operatorname{sol}_{i}(S)}{3},
$$

where

$$
\delta_{o c t}=\frac{-3 \pi+12 \arccos (1 / \sqrt{3})}{2 \sqrt{2}} \approx 0.72 .
$$


We let $a_{0}=a\left(y_{1}, y_{2}, y_{3}, y_{4}, y_{5}, y_{6}\right)$, where $a$ is the function defined in (8.4.1). We also let $a_{1}, a_{2}$, and $a_{3}$ be the functions $a$ for the vertices denoted $v_{1}, v_{2}$, and $v_{3}$ in Diagram 8.1.2. For example, $a_{1}=a\left(y_{1}, y_{5}, y_{6}, y_{4}, y_{2}, y_{3}\right)$. Set $t=\sqrt{\Delta} / 2$. By the results of Sections 8.1 and 8.4 , we find that $\Gamma(S)=-\delta_{\text {oct }} t / 6+\sum_{0}^{3} \frac{2}{3} \arctan \left(t / a_{i}\right)$. Recall that $a_{i}>0$ on a cell $C$. We wish to give an elementary upper bound of $\Gamma$ on $C$. Set, for $t \geq 0$ and $a=\left(a_{0}, a_{1}, a_{2}, a_{3}\right) \in \mathbb{R}_{+}^{4}$,

$$
\gamma(t, a):=\frac{-\delta_{o c t} t}{6}+\sum_{i=0}^{3} \frac{2}{3} \arctan \left(\frac{t}{a_{i}}\right) .
$$

The partial derivative of $\gamma$ with respect to $a_{i}$ is $-2 t /\left(3\left(t^{2}+a_{i}^{2}\right)\right) \leq 0$, so $\gamma(t, a) \leq$ $\gamma\left(t, a^{-}\right)$, where $a^{-}=\left(a_{0}^{-}, a_{1}^{-}, a_{2}^{-}, a_{3}^{-}\right)$is a lower bound of $a$ on $C$, as determined by Section 8.4. We study $\gamma(t)=\gamma\left(t, a^{-}\right)$as a function of $t$ to obtain an upper bound on $\Gamma(S)$. We note that

$$
\gamma^{\prime}(t)=\frac{-\delta_{o c t}}{6}+\frac{2}{3} \sum_{i=0}^{3} \frac{a_{i}}{\left(t^{2}+a_{i}^{2}\right)} \quad \text { and } \quad \gamma^{\prime \prime}(t)=-\frac{4}{3} \sum \frac{a_{i} t}{\left(t^{2}+a_{i}^{2}\right)^{2}} \leq 0 .
$$

Upper and lower bounds on $t$ are known from Section 8.1. An upper bound on $\gamma$ for $t$ in $\left[t_{\min }, t_{\max }\right]$ is the maximum of $\ell\left(t_{\min }\right)$ and $\ell\left(t_{\max }\right)$, where $\ell$ is the tangent to $\gamma$ at any point in $\left[t_{\min }, t_{\max }\right]$.

\subsection{Voronoi cells}

We assume in this section that the simplex $S$ has the property that the circumcenter of each of the three faces with vertex at the origin lies in the cone at the origin over the face. This condition is automatically satisfied if these three faces are acute triangles. In particular, it is satisfied for a quasi-regular tetrahedron.

When the cone over a quasi-regular tetrahedron $S$ contains the circumcenter of $S$, Section 2 sets vor $(S)=-4 \delta_{o c t} \operatorname{vol}\left(\hat{S}_{0}\right)+4 \operatorname{sol}(S) / 3$, where $\hat{S}_{0}$ is the intersection of $S$ with a Voronoi cell at the origin. Otherwise, $\operatorname{vor}(S)$ is defined as an analytic continuation. This section gives formulas for $\operatorname{vol}\left(\hat{S}_{0}\right)$.

As usual, set $S=S\left(y_{1}, \ldots, y_{6}\right)$ and $x_{i}=y_{i}^{2}$. Suppose at first that the circumcenter of $S$ is contained in the cone over $S$. The polyhedron $\hat{S}_{0}$ breaks into six pieces, called the Rogers simplices. A Rogers simplex is the convex hull of the origin, the midpoint of an edge (the first, second, or third edge), the circumcenter of a face along the given edge, and the circumcenter of $S$. Each Rogers simplex has the form

$$
R=R(a, b, c):=S\left(a, b, c,\left(c^{2}-b^{2}\right)^{1 / 2},\left(c^{2}-a^{2}\right)^{1 / 2},\left(b^{2}-a^{2}\right)^{1 / 2}\right)
$$

for some $1 \leq a \leq b \leq c$. Here $a$ is the half-length of an edge, $b$ is the circumradius of a face, and $c$ is the circumradius of the Delaunay simplex.

The volume is vol $(R(a, b, c))=a\left(b^{2}-a^{2}\right)^{1 / 2}\left(c^{2}-b^{2}\right)^{1 / 2} / 6$. The density $\delta(a, b, c)$ of $R=R(a, b, c)$ is defined as the ratio of the volume of the intersection of $R$ with a unit ball at the origin to the volume of $R$. It follows from the definitions that

$$
\operatorname{vor}(S)=\sum 4 \operatorname{vol}(R(a, b, c))\left(-\delta_{o c t}+\delta(a, b, c)\right),
$$


where $c$ is the circumradius $\operatorname{rad}(S)$, and $(a, b)$ runs over the six pairs

$$
\begin{array}{ll}
\left(\frac{y_{1}}{2}, \eta\left(y_{1}, y_{2}, y_{6}\right)\right), & \left(\frac{y_{2}}{2}, \eta\left(y_{1}, y_{2}, y_{6}\right)\right), \\
\left(\frac{y_{2}}{2}, \eta\left(y_{2}, y_{3}, y_{4}\right)\right), & \left(\frac{y_{3}}{2}, \eta\left(y_{2}, y_{3}, y_{4}\right)\right), \\
\left(\frac{y_{3}}{2}, \eta\left(y_{3}, y_{1}, y_{5}\right)\right), & \left(\frac{y_{1}}{2}, \eta\left(y_{3}, y_{1}, y_{5}\right)\right) .
\end{array}
$$

Upper and lower bounds on $\operatorname{vol}(R(a, b, c))$ follow without difficult from upper and lower bounds on $a, b$, and $c$. Thus, an upper bound on $\delta(a, b, c)$ leads to an upper bound on $\operatorname{vor}(S)$. The next lemma, which is due to Rogers, gives a good upper bound [R].

Lemma 8.6.2. The density $\delta(a, b, c)$ is monotonically decreasing in each variable for $1<a<b<c$.

Proof. Let $1 \leq a_{1} \leq b_{1} \leq c_{1}, 1 \leq a_{2} \leq b_{2} \leq c_{2}, a_{2} \leq a_{1}, b_{2} \leq b_{1}, c_{2} \leq c_{1}$. The points of $R\left(a_{1}, b_{1}, c_{1}\right)$ are realized geometrically by linear combinations

$$
\mathbf{s}_{1}=\lambda_{1}\left(a_{1}, 0,0\right)+\lambda_{2}\left(a_{1},\left(b_{1}^{2}-a_{1}^{2}\right)^{1 / 2}, 0\right)+\lambda_{3}\left(a_{1},\left(b_{1}^{2}-a_{1}^{2}\right)^{1 / 2},\left(c_{1}^{2}-b_{1}^{2}\right)^{1 / 2}\right),
$$

where $\lambda_{1}, \lambda_{2}, \lambda_{3} \geq 0$ and $\lambda_{1}+\lambda_{2}+\lambda_{3} \leq 1$. The points of $R\left(a_{2}, b_{2}, c_{2}\right)$ are realized geometrically by linear combinations

$$
\mathbf{s}_{2}=\lambda_{1}\left(a_{2}, 0,0\right)+\lambda_{2}\left(a_{2},\left(b_{2}^{2}-a_{2}^{2}\right)^{1 / 2}, 0\right)+\lambda_{3}\left(a_{2},\left(b_{2}^{2}-a_{2}^{2}\right)^{1 / 2},\left(c_{2}^{2}-b_{2}^{2}\right)^{1 / 2}\right),
$$

with the same restrictions on $\lambda_{i}$. Then

$$
\left|\mathbf{s}_{1}\right|^{2}-\left|\mathbf{s}_{2}\right|^{2}=\lambda_{1}\left(\lambda_{1}+2 \lambda_{2}+2 \lambda_{3}\right)\left(a_{1}^{2}-a_{2}^{2}\right)+\lambda_{2}\left(\lambda_{2}+2 \lambda_{3}\right)\left(b_{1}^{2}-b_{2}^{2}\right)+\lambda_{3}^{2}\left(c_{1}^{2}-c_{2}^{2}\right) .
$$

So $\left|s_{1}\right|^{2} \geq\left|s_{2}\right|^{2}$. This means that the linear transformation $s_{1} \mapsto s_{2}$ that carries the simplex $S_{1}$ to $S_{2}$ moves points of the simplex $S_{1}$ closer to the origin. In particular, the linear transformation carries the part in $S_{1}$ of the unit ball at the origin into the unit ball. This means that the density of $R\left(a_{1}, b_{1}, c_{1}\right)$ is at most that of $R\left(a_{2}, b_{2}, c_{2}\right)$.

If the circumcenter of $S$ is not in the cone over $S$, then the analytic continuation gives

$$
\operatorname{vor}(S)=\sum_{R} 4 \varepsilon_{R} \operatorname{vol}(R(a, b, c))\left(-\delta_{o c t}+\delta(a, b, c)\right),
$$

where $\varepsilon_{R}=1$ if the face of the Delaunay simplex $S$ corresponding to $R$ has positive orientation, and $\varepsilon_{R}=-1$ otherwise. (The face of $S$ "corresponding" to $R(a, b, c)$ is the one used to compute the circumradius $b$.)

8.6.3. A calculation based on the explicit coordinates of $S$ and its circumcenter given in Lemma 8.1.4 shows that

$$
\varepsilon_{R} \operatorname{vol}\left(R\left(\frac{y_{1}}{2}, \eta\left(y_{1}, y_{2}, y_{6}\right), \operatorname{rad}(S)\right)\right)=\frac{x_{1}\left(x_{2}+x_{6}-x_{1}\right) \chi\left(x_{4}, x_{5}, x_{3}, x_{1}, x_{2}, x_{6}\right)}{48 u\left(x_{1}, x_{2}, x_{6}\right) \Delta\left(x_{1}, \ldots, x_{6}\right)^{1 / 2}} .
$$


If the circumcenter of $S$ is not contained in $S$, then the same formula holds by analytic continuation. By definition, $\varepsilon_{R}=-1$ exactly when the function $\chi$ is negative. Although this formula is more explicit than the earlier formula, it tends to give weaker estimates of $\operatorname{vor}(S)$ and was not used in the calculations in Section 9.

8.6.4. There is another approximation to $\operatorname{vor}(S)$ that will be useful. Set $S_{y}=$ $S(2,2,2, y, y, y)$. (We hope there is no confusion with the previous notation $S_{i}$.) For $1 \leq a \leq b \leq c$, let $\operatorname{vol}(R(a, b, c))=a\left(\left(b^{2}-a^{2}\right)\left(c^{2}-b^{2}\right)\right)^{1 / 2} / 6$ be as above. Set $r(a)=\operatorname{vol}(R(a, \eta(2,2,2 a), 1.41))$.

Lemma 8.6.5. Assume that the circumradius of a quasi-regular tetrahedron $S$ is at least 1.41 , and that $6 \leq y_{1}+y_{2}+y_{3} \leq 6.3$. Set $a=\left(y_{1}+y_{2}+y_{3}-4\right) / 2$. Pick y to satisfy $\operatorname{sol}\left(S_{y}\right)=\operatorname{sol}(S)$. Then

$$
\operatorname{vor}(S) \leq \operatorname{vor}\left(S_{y}\right)-8 \delta_{o c t}\left(1-\frac{1}{a^{3}}\right) r(a)
$$

Proof. If $S$ is any quasi-regular tetrahedron, let $S_{\text {tan }}$ be the simplex defining the "tangent" Voronoi cell, that is, $S_{\text {tan }}$ is the simplex with the same origin that cuts out the same spherical triangle as $S$ on the unit sphere, but that satisfies $y_{1}=y_{2}=y_{3}=2$. The lengths of the fourth, fifth, and sixth edges of $S_{\text {tan }}$ are between $\sqrt{8-2(2.3)}=\sqrt{3.4}$ and 2.51 . The faces of $S_{\tan }$ are acute triangles. A calculation similar to the proof in paragraph 8.2.5, based on $\chi\left(3.4,3.4,4,4,4,2.51^{2}\right)>0$, shows that the circumcenter of $S_{\tan }$ is contained in the cone over $S_{\text {tan }}$.

Since $S_{\text {tan }}$ is obtained by "truncating" $S$, we observe that $\operatorname{vor}\left(S_{\text {tan }}\right)-4 \delta_{o c t} \operatorname{vol}\left(\hat{S} \backslash \hat{S}_{\text {tan }}\right)$, where $\hat{S}$ and $\hat{S}_{\text {tan }}$ are the pieces of Voronoi cells denoted $\hat{S}_{0}$ in Section 2 for $S$ and $S_{\text {tan }}$, respectively. By a convexity result of Fejes Tóth, $\operatorname{vor}\left(S_{\text {tan }}\right) \leq \operatorname{vor}\left(S_{y}\right)[\mathrm{FT}, \mathrm{p} .125]$. (This inequality relies on the fact that the circumcenter of $S_{\mathrm{tan}}$ is contained in the cone over $S_{\text {tan. }}$.)

Let $a_{1}$ be the half-length of the first, second, or third edge. Since $\operatorname{vol}\left(R\left(a_{1}, b, c\right)\right)$ is increasing in $c$, we obtain a lower bound on $\operatorname{vol}\left(R\left(a_{1}, b, c\right)\right)$ for $c=1.41$, the lower bound on the circumradius of $S$. The function $\operatorname{vol}\left(R\left(a_{1}, b, 1.41\right)\right)$, considered as a function of $b$, has at most one critical point in $\left[a_{1}, 1.41\right]$ and it is always a local maximum (by a second derivative test). Thus,

(8.6.6) $\operatorname{vol}\left(R\left(a_{1}, b, c\right)\right) \geq \min \left(\operatorname{vol}\left(R\left(a_{1}, b_{\min }, 1.41\right)\right), \operatorname{vol}\left(R\left(a_{1}, b_{\max }, 1.41\right)\right)\right)$,

where $b_{\min }$ and $b_{\max }$ are upper and lower bounds on $b \in\left[a_{1}, 1.41\right]$. A lower bound on $b$ is $\eta\left(2,2,2 a_{1}\right)$, which means that $\operatorname{vol}\left(R\left(a_{1}, b_{\min }, 1.41\right)\right)$ may be replaced with $r\left(a_{1}\right)$ in inequality (8.6.6). By Heron's formula, the function $\eta(a, b, c)$, for acute triangles, is convex in pairs of variables:

$$
\eta_{a a} \eta_{b b}-\eta_{a b}^{2}=\frac{\eta^{6}\left(a^{2}+b^{2}-c^{2}\right)\left(a^{2}-b^{2}+c^{2}\right)\left(-a^{2}+b^{2}+c^{2}\right)\left(a^{2}+b^{2}+c^{2}\right)}{a^{6} b^{6} c^{4}}>0 .
$$

Thus, an upper bound on $b_{\max }$ is $\eta(2,2.51,2 a)$, where $a=\left(y_{1}+y_{2}+y_{3}-4\right) / 2$. This means that

$$
\operatorname{vol}\left(R\left(\left(a_{1}, b_{\max }, 1.41\right)\right)\right)
$$


may be replaced with the function $\operatorname{vol}\left(R\left(a_{1}, \eta(2,2.51,2 a), 1.41\right)\right)$ in inequality (8.6.6). Now $1 \leq a_{1} \leq a$, and $\operatorname{vol}\left(R\left(a_{1}, b, c\right)\right)$ is decreasing in the first variable (for $1 \leq a_{1}$ and $b \leq \sqrt{2}$ ), so we may use the lower bound

$$
\operatorname{vol}(R(a, \eta(2,2.51,2 a), 1.41))
$$

instead. By Calculations 9.20.2 and 9.20.3, we conclude that vol $\left(R\left(a_{1}, b, c\right)\right) \geq r(a)$. This lower bound is valid for each Rogers simplex. The volume of $\hat{S} \backslash \hat{S}_{\text {tan }}$ is then at least

$$
\left(\left(1-\frac{8}{y_{1}^{3}}\right)+\left(1-\frac{8}{y_{2}^{3}}\right)+\left(1-\frac{8}{y_{3}^{3}}\right)\right) 2 r(a) .
$$

The concavity of $1-8 / y^{3}$ gives $\sum_{i=1}^{3}\left(1-8 / y_{i}^{3}\right) \geq 1-1 / a^{3}$. We have established that

$$
\operatorname{vol}\left(\hat{S} \backslash \hat{S}_{\mathrm{tan}}\right) \geq\left(1-\frac{1}{a^{3}}\right) 2 r(a) .
$$

The result follows.

8.6.7. We conclude our discussion of Voronoi cells with a few additional comments about the case in which analytic continuation is used to define $\operatorname{vor}(S)$, with $S$ a quasiregular tetrahedron. Assume the circumcenter $c$ of $S$ lies outside $S$ and that the face $T$ of $S$ with negative orientation is the one bounded by the first, second, and sixth edges. It follows from Section 3 that $y_{1}, y_{2}, y_{6} \in[2.3,2.51]$ and $y_{3}, y_{4}, y_{5} \in[2,2.15]$.

Let $p_{1}$ (resp. $p_{2}$ ) be the point on $T$ equidistant from the origin, $v_{3}$, and $v_{1}$ (resp. $v_{2}$ ).

$$
\left|p_{1}\right|^{2}=\frac{x_{1}}{4}+\frac{x_{1} u\left(x_{1}, x_{2}, x_{6}\right)\left(-x_{1}+x_{3}+x_{5}\right)^{2}}{4\left(\partial \Delta / \partial x_{4}\right)^{2}} .
$$

Let $p_{0}$ be the circumcenter of $T$ (see Diagram 8.6.8).

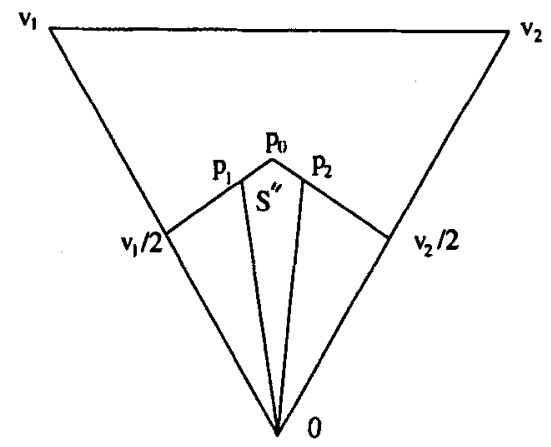

Diagram 8.6.8

$\varepsilon_{R}=-1$ for the Rogers simplex with vertices the origin, $p_{0}, c$, and $v_{1} / 2$. It lies outside $S$. The other Rogers simplex along the first edge has $\varepsilon_{R}=1$, so that the part 
common to both of these Rogers simplices cancels in the definition of vor $(S)$. This means that $\operatorname{vor}(S)$ becomes the sum of the usual contributions from the two Rogers simplices along the third edge,

$$
4 \operatorname{vol}(R(a, b, c))\left(-\delta_{o c t}+\delta(a, b, c)\right)
$$

for $(a, b, c)=\left(y_{1} / 2, \eta\left(y_{1}, y_{3}, y_{5}\right),\left|p_{1}\right|\right)$ and $\left(y_{2} / 2, \eta\left(y_{2}, y_{3}, y_{4}\right),\left|p_{2}\right|\right)$, and

$$
v\left(S^{\prime \prime}\right):=4 \delta_{o c t} \operatorname{vol}\left(S^{\prime \prime}\right)-\frac{4 \operatorname{sol}\left(S^{\prime \prime}\right)}{3},
$$

where $S^{\prime \prime}$ is the convex hull of $0, p_{0}, p_{1}, p_{2}$, and $c$.

We claim that in any cell satisfying the constraints given above, $\left|p_{1}\right|$ is minimized by making $y_{1}, y_{2}, y_{6}$ as large as possible and $y_{3}, y_{4}, y_{5}$ as small as possible. To show this, the derivatives have to be written explicitly from the formula for $\left|p_{1}\right|^{2}$ given above. The partial derivatives with respect to $x_{2}, \ldots, x_{6}$ factor into products of the polynomials $\partial \Delta / \partial x_{i}$, for $i=3,4,5, x_{1},\left(x_{3}+x_{5}-x_{1}\right), u\left(x_{1}, x_{2}, x_{6}\right),\left(-x_{1} x_{4}+x_{2} x_{5}+x_{1} x_{6}-x_{5} x_{6}\right)$, and $\left(x_{1} x_{2}-x_{2} x_{3}-x_{1} x_{4}+x_{3} x_{6}\right)$. The signs of these polynomials are easily determined. The partial with respect to $x_{1}$ is complicated (the numerator has 88 terms), and we had to resort to the method of subdivision to determine its sign. We omit the details.

To complete our estimate, we describe an upper bound on $v\left(S^{\prime \prime}\right)$. The ratio sol $\left(S^{\prime \prime}\right) /$ $\left(3 \mathrm{vol}\left(S^{\prime \prime}\right)\right)$ is at least $1 / \operatorname{rad}(S)^{3}$, because $S^{\prime \prime}$ is contained in a sphere of $\operatorname{radius} \operatorname{rad}(S)$, centered at the origin. We have

$$
\frac{\operatorname{vol}\left(S^{\prime \prime}\right)}{2} \leq \frac{1}{6}\left|p_{0}-p_{1}\right|\left|c-p_{0}\right|\left|p_{0}\right|
$$

because the convex hull of $0, p_{0}, p_{1}$, and $c$, which is one side of $S^{\prime \prime}$, is a pyramid with base the right triangle $\left(p_{1}, p_{0}, c\right)$ and height at most $\left|p_{0}\right|=\eta\left(y_{1}, y_{2}, y_{6}\right)$. Of course, $\left|c-p_{0}\right|^{2}=\operatorname{rad}(S)^{2}-\eta\left(y_{1}, y_{2}, y_{6}\right)^{2}$. We now have a bound on $v\left(S^{\prime \prime}\right)$ in terms of quantities that have been studied in Section 8 , if we rely on the bound $\left|p_{0}-p_{1}\right| \leq 0.1381$. Write $p_{1}=p_{1}\left(y_{1}, \ldots, y_{6}\right)$. This bound is obtained from the following inequalities:

$$
\begin{aligned}
\left|p_{0}-p_{1}\right|= & \left(\eta\left(y_{1}, y_{2}, y_{6}\right)^{2}-\frac{x_{1}}{4}\right)^{1 / 2}-\left(\left|p_{1}\right|^{2}-\frac{x_{1}}{4}\right)^{1 / 2} \\
\leq & \left(\eta\left(y_{1}, 2.51,2.51\right)^{2}-\frac{x_{1}}{4}\right)^{1 / 2}-\left(\left|p_{1}\left(y_{1}, 2.51,2,2,2,2.51\right)\right|^{2}-\frac{x_{1}}{4}\right)^{1 / 2} \\
\leq & \left(\eta(2.51,2.51,2.51)^{2}-\frac{2.51^{2}}{4}\right)^{1 / 2} \\
& -\left(\left|p_{1}(2.51,2.51,2,2,2,2.51)\right|^{2}-\frac{2.51^{2}}{4}\right)^{1 / 2} \\
< & 0.1381 .
\end{aligned}
$$

The inequality that replaces $y_{1}$ with 2.51 results from Calculation 9.21 .

\subsection{A Final Reduction}

Let $S$ be a Delaunay simplex. Suppose that the lengths of the edges $y_{1}, y_{5}$, and $y_{6}$ are greater than 2 . Let $S^{\prime}$ be a simplex formed by contracting the vertex joining edges 1,5 , 
and 6 along the first edge by a small amount. We assume that the lengths $y_{1}^{\prime}, y_{5}^{\prime}$, and $y_{6}^{\prime}$ of the new edges are still at least 2 and that the circumradius of $S^{\prime}$ is at most 2 , so that $S^{\prime}$ is a Delaunay simplex.

Proposition 8.7.1. $\Gamma\left(S^{\prime}\right)>\Gamma(S)$.

We write $\operatorname{sol}_{i}$, for $i=1,2,3$, for the solid angles at the three vertices $p_{1}, p_{2}$, and $p_{3}$ of $S$ terminating the edges 1,2 , and 3 . Let $p_{1}^{\prime}$ be the vertex terminating edge 1 of $S^{\prime}$. Similarly, we write $\operatorname{sol}_{i}^{\prime}$, for $i=1,2,3$, for the solid angles at the corresponding vertices of $S^{\prime}$. We set $\operatorname{vol}(V)=\operatorname{vol}(S)-\operatorname{vol}\left(S^{\prime}\right)$ and $w_{i}=\operatorname{sol}_{i}-\operatorname{sol}_{i}^{\prime}$. It follows directly from the construction of $S^{\prime}$ that $w_{2}$ and $w_{3}$ are positive. The dihedral angle $\alpha$ along the first edge is the same for $S$ and $S^{\prime}$. The angle $\beta_{i}$ of the triangle $\left(0, p_{1}, p_{i}\right)$ at $p_{1}$ is less than the angle $\beta_{i}^{\prime}$ of the triangle $\left(0, p_{1}^{\prime}, p_{i}\right)$ at $p_{1}^{\prime}$, for $i=2$, 3. It follows that $w_{1}$ is negative, since $-w_{1}$ is the area of the quadrilateral region of Diagram 8.7.2 on the unit sphere.

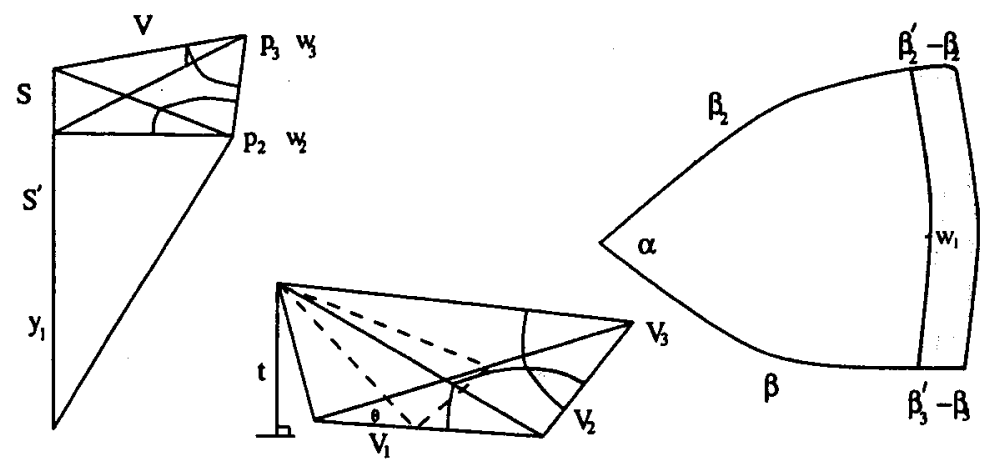

Diagram 8.7.2

Lemma 8.7.3. $\delta_{o c t} \operatorname{vol}(V)>w_{2} / 3+w_{3} / 3$.

The lemma immediately implies the proposition because $w_{1}<0$ and

$$
\Gamma\left(S^{\prime}\right)-\Gamma(S)=\frac{-w_{1}}{3}-\frac{w_{2}}{3}-\frac{w_{3}}{3}+\delta_{o c t} \operatorname{vol}(V) .
$$

Proof. Let $T^{\prime}$ be the face of $S^{\prime}$ with vertices $p_{1}^{\prime}, p_{2}$, and $p_{3}$. We consider $S^{\prime}$ as a function of $t$, where $t$ is the distance from $p_{1}$ to the plane containing $T^{\prime}$. (See Diagram 8.7.2.) It is enough to establish the lemma for $t$ infinitesimal.

As shown in Diagram 8.7.2, let $V_{1}$ be the pyramid formed by intersecting $V$ with the plane through $p_{1}$ that meets the fifth edge at distance $t_{0}=1.15$ from $p_{3}$ and the sixth edge at distance $t_{0}$ from $p_{2}$. Also, let the intersection of $V$ with a ball of radius $t_{0}$ centered at $p_{i}$ be denoted $V_{i}$, for $i=2,3$. For $t$ sufficiently small, the region $V_{1}$ is (essentially) disjoint from $V_{2}$ and $V_{3}$.

We claim that $\operatorname{vol}\left(V_{1}\right)>\operatorname{vol}\left(V_{2} \cap V_{3}\right)$. Let $\theta$ be the angle of $T^{\prime}$ subtended by the fifth and sixth edges of $S^{\prime}$. Then $\operatorname{vol}\left(V_{1}\right)=B t / 3$, where $B$ is the area of the intersection of 
$V_{1}$ and $T^{\prime}$ :

$$
B=\frac{\sin \theta\left(y_{5}^{\prime}-t_{0}\right)\left(y_{6}^{\prime}-t_{0}\right)}{2} .
$$

Since $S^{\prime}$ is a Delaunay simplex, the estimates $\pi / 6 \leq \theta \leq 2 \pi / 3$ from [H1, 2.3] hold. In particular, $\sin \theta \geq 0.5$, so $B \geq 0.25(2-1.15)^{2}$, and vol $\left(V_{1}\right)>0.06 t$.

If $\operatorname{vol}\left(V_{2} \cap V_{3}\right)$ is nonempty, the fourth edge of $S$ must have length less than $2 t_{0}$. The dihedral angle $\alpha^{\prime}$ of $V$ along the fourth edge is then less than the tangent of the angle, which is at most $t+O\left(t^{2}\right)$, in Landau's notation. As in [H1, 5], we obtain the estimate

$$
\operatorname{vol}\left(V_{2} \cap V_{3}\right) \leq \alpha^{\prime} \int_{1}^{t_{0}} t_{0}^{2}-t^{2} d t=\frac{\left(t_{0}-1\right)^{2}\left(2 t_{0}+1\right) \alpha^{\prime}}{3}<0.025 t+O\left(t^{2}\right)
$$

This establishes the claim.

Thus, for $t$ sufficiently small

$$
\begin{aligned}
\delta_{o c t} \operatorname{vol}(V) & \geq \delta_{o c t}\left(\operatorname{vol}\left(V_{1}\right)+\operatorname{vol}\left(V_{2}\right)+\operatorname{vol}\left(V_{3}\right)-\operatorname{vol}\left(V_{2} \cap V_{3}\right)\right) \\
& >\left(\delta_{o c t} t_{0}^{3}\right)\left(\frac{w_{2}}{3}+\frac{w_{3}}{3}\right)>1.09\left(\frac{w_{2}}{3}+\frac{w_{3}}{3}\right) .
\end{aligned}
$$

\section{Floating-Point Calculations}

This section describes various inequalities that have been established by the method of subdivision on SUN workstations. The full source code (in $\mathrm{C}++$ ) for these calculations is available [H6].

Floating-point operations on computers are subject to round-off errors, making many machine computations unreliable. Methods of interval arithmetic give users control over round-off errors [AH]. These methods may be reliably implemented on machines that allow arithmetic with directed rounding, for example, those conforming to the IEEE/ANSI standard 754 [W], [IEEE], [P].

Interval arithmetic produces an interval in the real line that is guaranteed to contain the result of an arithmetic operation. As the round-off errors accumulate, the interval grows wider, and the correct answer remains trapped in the interval. Apart from the risk of compiler errors and defective hardware, a bound established by interval arithmetic is as reliable as a result established by integer arithmetic on a computer. We have used interval arithmetic wherever computer precision is a potential issue (Calculations 9.1-9.19, in particular).

Every inequality of this section has been reduced to a finite number of inequalities of the form $r\left(x_{0}\right)<0$, where $r$ is a rational function of $x \in \mathbb{R}^{n}$ and $x_{0}$ is a given element in $\mathbb{R}^{n}$. To evaluate each rational expression, interval arithmetic is used to obtain an interval $Y$ containing $r\left(x_{0}\right)$. The stronger inequality, $y<0$ for all $y \in Y$, which may be verified by computer, implies that $r\left(x_{0}\right)<0$.

To reduce the calculations to rational expressions $r\left(x_{0}\right)$, rational approximations to the functions $\sqrt{x}, \arctan (x)$, and $\arccos (x)$ with explicit error bounds are required. These were obtained from [H7]. Reliable approximations to various constants (such as $\pi, \sqrt{2}$, and $\delta_{o c t}$ ) with explicit error bounds are also required. These were obtained in Mathematica and were double checked against Maple. 
Let $S=S\left(y_{1}, \ldots, y_{6}\right)$ be a quasi-regular tetrahedron. We label the indices as in Diagram 8.1.2. Let $\Gamma=\Gamma\left(S\left(y_{1}, \ldots, y_{6}\right)\right)$ be the compression. We also let dih $=$ $\operatorname{dih}\left(S\left(y_{1}, \ldots, y_{6}\right)\right)$ be the dihedral angle along the first edge, and let sol $=$ sol $\left(S\left(y_{1}, \ldots, y_{6}\right)\right)$ be the solid angle at the origin, that is, the solid angle formed by the first, second, and third edges $\left(y_{1}, y_{2}, y_{3}\right)$ of $S$.

All of the following inequalities are to be considered as inequalities of analytic functions of $y_{1}, \ldots, y_{6}$. Although each of the calculations is expressed as an inequality between functions of six variables, Proposition 8.7 .1 has been invoked repeatedly to reduce the number of variables to three or four. For instance, suppose that we wish to establish $I($ sol, $\Gamma)<0$, where $I($ sol, $\Gamma)$ is an expression in $\Gamma\left(S\left(y_{1}, \ldots, y_{6}\right)\right)$ and $\operatorname{sol}\left(S\left(y_{1}, \ldots, y_{6}\right)\right)$. Invoking Proposition 8.7.1 three times, we may assume that the vertices marked $v_{1}, v_{2}$, and $v_{3}$ in Diagram 8.1.2 each terminate an edge of minimal length. It is then sufficient to establish the inequality in seven situations of smaller dimension; that is, we may assume the edges $i \in I$ have minimal length, where $I$ is one of

$$
\{1,4\},\{2,5\},\{3,6\},\{4,5\},\{4,6\},\{5,6\},\{1,2,3\} \text {. }
$$

Similarly, for an inequality in $\Gamma$ and dihedral angle, we reduce to the seven cases

$$
I=\{1,4\},\{2,5\},\{3,6\},\{1,2,3\},\{1,5,6\},\{3,4,5\},\{2,4,6\} .
$$

The first two calculations are inequalities of the compression $\Gamma$ of a quasi-regular tetrahedron.

Calculation 9.1. $\Gamma \leq 1 \mathrm{pt}$.

This first inequality and Calculation 9.3 are the only ones that are not strict inequalities. Set $S_{0}=S(2,2,2,2,2,2)$. By definition, $\Gamma\left(S_{0}\right)=1 \mathrm{pt}$. We must give a direct proof that $S_{0}$ gives the maximum in an explicit neighborhood. Then we use the method of subdivision to bound $\Gamma$ away from $1 p t$ outside the given neighborhood. An infinitesimal version of the following result is proved in [H1].

Lemma 9.1.1. If $y_{i} \in[2,2.06]$, for $i=1, \ldots, 6$, then $\Gamma\left(S\left(y_{1}, \ldots, y_{6}\right)\right) \leq \Gamma\left(S_{0}\right)$, with equality if and only if $S\left(y_{1}, \ldots, y_{6}\right)=S_{0}$.

Set $a_{00}=a(2,2,2,2,2,2)=20, \Delta_{0}=\Delta\left(2^{2}, 2^{2}, 2^{2}, 2^{2}, 2^{2}, 2^{2}\right), t_{0}=\sqrt{\Delta_{0}} / 2=$ $4 \sqrt{2}$, and $b_{0}=\frac{2}{3}\left(1+t_{0}^{2} / a_{00}^{2}\right)^{-1}=50 / 81$. Set $f=\max _{i}\left(y_{i}-2\right) \leq 0.06$ and $a^{-}=$ $a(2,2,2,2+f, 2+f, 2+f)=20-12 f-3 f^{2}$. As in Section 8, we set $t=\sqrt{\Delta} / 2$. We have $\arctan (x) \leq \arctan \left(x_{0}\right)+\left(x-x_{0}\right) /\left(1+x_{0}^{2}\right)$, if $x, x_{0} \geq 0$. We verify below that $\Delta \geq \Delta_{0}$ under the restrictions given above. Then

$$
\begin{aligned}
\Gamma(S) & =-\frac{\delta_{\text {oct } t}}{6}+\frac{2}{3} \sum_{i=0}^{3} \arctan \left(\frac{t}{a_{i}}\right) \\
& \leq \Gamma\left(S_{0}\right)-\frac{\delta_{\text {oct }}\left(t-t_{0}\right)}{6}+b_{0} \sum_{i=0}^{3}\left(\frac{t}{a_{i}}-\frac{t_{0}}{a_{00}}\right)
\end{aligned}
$$




$$
\begin{aligned}
= & \Gamma\left(S_{0}\right)-\frac{\delta_{o c t}\left(\Delta-\Delta_{0}\right)}{24\left(t+t_{0}\right)}+b_{0} \sum_{i=0}^{3} \frac{t-t_{0}}{a_{i}} \\
& +\frac{b_{0} t_{0}}{a_{00}^{2}} \sum_{i=0}^{3}\left(a_{00}-a_{i}\right)+\frac{b_{0} t_{0}}{a_{0}^{2}} \sum_{i=0}^{3} \frac{\left(a_{00}-a_{i}\right)^{2}}{a_{i}} \\
\leq & \Gamma\left(S_{0}\right)+\frac{\left(\Delta-\Delta_{0}\right)}{t+t_{0}}\left(-\frac{\delta_{o c t}}{24}-\frac{b_{0}}{a^{-}}\right)+\frac{b_{0} t_{0}}{a_{00}^{2}} \sum_{i=0}^{3}\left(a_{00}-a_{i}\right) \\
& +\frac{b_{0} t_{0}}{a_{0}^{2} a^{-}} \sum_{i=0}^{3}\left(a_{00}-a_{i}\right)^{2} .
\end{aligned}
$$

Set $c_{0}=-\delta_{\text {oct }} / 24+b_{0} / a^{-}>0$. Write $y_{i}=2+f_{i}$, with $0 \leq f_{i} \leq f$. Set $x_{i}=$ $4+e_{i}=y_{i}^{2}=4+4 f_{i}+f_{i}^{2}$. Then $f_{i} \leq e_{i} / 4$, for $i=1, \ldots, 6$. Set $e=4 f+f^{2}$. Set $\bar{\Delta}\left(e_{1}, \ldots, e_{6}\right)=\Delta\left(x_{1}, \ldots, x_{6}\right)$. We find that $\partial^{2} \tilde{\Delta} / \partial e_{2} \partial e_{1}=4+e_{4}+e_{5}-e_{6}>0$ and similarly that $\partial^{2} \tilde{\Delta} / \partial e_{i} \partial e_{1}>0$, for $i=3,5,6$. So $\partial \tilde{\Delta} / \partial e_{\mathrm{J}}$ is minimized by taking $e_{2}=e_{3}=e_{5}=e_{6}=0$, and this partial derivative is at least

$$
\frac{\partial \tilde{\Delta}}{\partial e_{1}}\left(e_{1}, 0,0, e_{4}, 0,0\right)=16-8 e_{1}-2 e_{1} e_{4}-e_{4}^{2} \geq 16-8 e-3 e^{2}>0 .
$$

Thus $\Delta \geq \Delta_{0}$. The partial derivative $\partial \tilde{\Delta} / \partial e_{1}$ is at most

$$
\begin{aligned}
\frac{\partial \tilde{\Delta}}{\partial e_{1}}\left(e_{1}, e, e, e_{4}, e, e\right) & =16+16 e-8 e_{1}+4 e e_{4}-2 e_{1} e_{4}-e_{4}^{2} \leq 16+16 e+4 e e_{4}-e_{4}^{2} \\
& \leq 16+16 e+3 e^{2} .
\end{aligned}
$$

So $\Delta-\Delta_{0} \leq\left(16+16 e+3 e^{2}\right)\left(e_{1}+\cdots+e_{6}\right)$.

We expand $\sum_{i=0}^{3}\left(a_{i}-a_{00}\right)=5\left(e_{1}+\cdots+e_{6}\right)+h_{1}+h_{2}+h_{3}$ as a sum of homogeneous polynomials $h_{i}$ of degree $i$ in $f_{1}, \ldots, f_{6}$. A calculation shows that $h_{1}=0$. Also $h_{2}$ is quadratic in each variable $f_{i}$ with negative leading coefficient, so $h_{2}$ attains its minimum at an extreme point of the cube $[0, f]^{6}$. A calculation then shows that $h_{2} \geq-14 f^{2}$ on $[0, f]^{6}$. By discarding all the positive terms of $h_{3}$, we find that

$$
h_{3} \geq-f_{1}^{2} f_{4}-f_{1} f_{4}^{2}-f_{2}^{2} f_{5}-f_{2} f_{5}^{2}-f_{3}^{2} f_{6}-f_{3} f_{6}^{2} \geq-6 f^{3} .
$$

Thus, $\sum_{i=0}^{3}\left(a_{i}-a_{00}\right) \geq 5\left(e_{1}+\cdots+e_{6}\right)-14 f^{2}-6 f^{3}$. Since $e=\max \left(e_{i}\right)$, we find that $f \leq e / 4 \leq\left(e_{1}+\cdots+e_{6}\right) / 4$. This gives

$$
\sum_{i=0}^{3}\left(a_{00}-a_{i}\right) \leq\left(e_{1}+\cdots+e_{6}\right)\left(-5+\frac{7 f}{2}+\frac{3 f^{2}}{2}\right) .
$$

Similarly, we expand $\sum_{i=0}^{3}\left(a_{00}-a_{i}\right)^{2}$ as a polynomial in $f_{1}, \ldots, f_{6}$. To obtain an upper bound, we discard all the negative terms of the polynomial and evaluate all the positive terms at $\left(f_{1}, \ldots, f_{6}\right)=(f, \ldots, f)$. This gives

$$
\begin{aligned}
\sum_{i=0}^{3}\left(a_{00}-a_{i}\right)^{2} & \leq 4944 f^{2}+7296 f^{3}+3684 f^{4}+828 f^{5}+73 f^{6} \\
& \leq \frac{1}{4}\left(e_{1}+\cdots+e_{6}\right)\left(4944 f+7296 f^{2}+3684 f^{3}+828 f^{4}+73 f^{5}\right) .
\end{aligned}
$$


We now insert these estimates back into inequality (9.1.2). This gives

$$
\begin{aligned}
\frac{\Gamma(S)-\Gamma\left(S_{0}\right)}{\left(e_{1}+\cdots+e_{6}\right)} \leq & \frac{c_{0}}{2 t_{0}}\left(16+16 e+3 e^{2}\right)+\frac{b_{0} t_{0}}{a_{00}^{2}}\left(-5+3.5 f+1.5 f^{2}\right) \\
& +\frac{b_{0} t_{0}}{4 a_{00}^{2} a^{-}}\left(4944 f+7296 f^{2}+3684 f^{3}+828 f^{4}+73 f^{5}\right) .
\end{aligned}
$$

The right-hand side of this inequality is a rational function of $f$. (Both $a^{-}$and $e$ depend on $f$.) Each of the three terms on the right-hand side is increasing in $f$. Therefore, the right-hand side reaches its maximum at $f=0.06$. Direct evaluation at $f=0.06$ gives $\Gamma(S)-\Gamma\left(S_{0}\right) \leq-0.00156\left(e_{1}+\cdots+e_{6}\right)$.

To verify Calculation 9.14 , the computer examined only 7 cells. Calculation 9.1 required 1899 cells. Calculation 9.6.1 required over 2 million cells. The number of cells required in the verification of the other inequalities falls between these extremes.

Calculation 9.2. $\Gamma<0.5 p t$, if $y_{1} \in[2.2,2.51]$.

The next several calculations are concerned with the relationship between the dihedral angle and the compression.

Calculation 9.3. $\operatorname{dih}\left(S\left(y_{1}, \ldots, y_{6}\right)\right) \geq \operatorname{dih}_{\min }:=\operatorname{dih}(S(2,2.51,2,2,2.51,2)) \approx$ 0.8639 .

Since this bound is realized by a simplex, we must carry out the appropriate local analysis in a neighborhood of $S(2,2.51,2,2,2.51,2)$.

Lemma 9.3.1. Suppose that $2 \leq y_{i} \leq 2.2$, for $i=1,3,6$, and that $2 \leq y_{i}=2.51$, for $i=2,4,5$. Then $\operatorname{dih}\left(S\left(y_{1}, \ldots, y_{6}\right)\right) \geq \operatorname{dih}_{\min }$.

Proof. This is an application of Lemma 8.3.1. By that lemma, $\operatorname{dih}(S)$ is increasing in $x_{4}$, so we fix $x_{4}=4$. The sign of $\partial \cos \operatorname{dih} / \partial x_{2}$ is the sign of $\partial \Delta / \partial x_{3}$, and a simple estimate based on the explicit formulas of Section 8 shows that $\partial \Delta / \partial x_{3}>0$ under the given constraints. Thus, we minimize $\operatorname{dih}(S)$ by setting $x_{2}=2.51^{2}$. By symmetry, we set $x_{5}=2.51^{2}$.

Now consider $\operatorname{dih}(S)$ as a function of $x_{1}, x_{3}$, and $x_{6}$. The sign of $\partial \cos \operatorname{dih} / \partial x_{3}$ is the sign of $\partial \Delta\left(x_{1}, 2.51^{2}, x_{3}, 2^{2}, 2.51^{2}, x_{6}\right) / \partial x_{2}$. The maximum of this partial (about -2.39593 ) is attained when $x_{1}, x_{3}$, and $x_{6}$ are as large as possible: $x_{1}=x_{3}=x_{6}=2.2^{2}$. So $\operatorname{dih}(S)$ is increasing in $x_{3}$. We take $x_{3}=4$, and by symmetry $x_{6}=4$.

We have

$$
\frac{\partial \cos \operatorname{dih}(S)}{\partial x_{1}}=\frac{2 t_{1}\left(x_{1}\right)}{u\left(x_{1}, x_{2}, x_{6}\right)^{3 / 2} u\left(x_{1}, x_{3}, x_{5}\right)^{3 / 2}},
$$

where $t_{1}\left(x_{1}\right) \approx 464.622-1865.14 x_{1}+326.954 x_{1}^{2}-92.9817 x_{1}^{3}+4 x_{1}^{4}$. An estimate of the derivative of $t_{1}(x)$ shows that $t_{1}(x)$ attains its maximum at $x_{1}=4$, and $t_{1}(4)<$ $-6691<0$. Thus $\operatorname{dih}(S)$ is minimized when $x_{1}=4$.

Calculation 9.4. $\Gamma<0.378979 \mathrm{dih}-0.410894$. 
Lemma 9.5. If $S_{1}, S_{2}, S_{3}$, and $S_{4}$ are any four tetrahedra such that $\operatorname{dih}\left(S_{1}\right)+\operatorname{dih}\left(S_{2}\right)+$ $\operatorname{dih}\left(S_{3}\right)+\operatorname{dih}\left(S_{4}\right) \geq 2 \pi$, then $\Gamma\left(S_{1}\right)+\cdots+\Gamma\left(S_{4}\right)<0.33 p t$.

This lemma is a consequence of the following three calculations, each established by interval arithmetic and the method of subdivision.

\section{Calculation 9.5.1.}

$$
\Gamma<-0.19145 \mathrm{dih}+0.2910494,
$$

provided $\operatorname{dih} \in[\operatorname{dih}(S(2,2,2,2,2,2)), 1.42068]$.

\section{Calculation 9.5.2.}

$$
\Gamma<-0.0965385 \mathrm{dih}+0.1562106,
$$

provided dih $\in[1.42068, \operatorname{dih}(S(2,2,2,2.51,2,2))]$.

\section{Calculation 9.5.3.}

$$
\Gamma<-0.19145 \mathrm{dih}+0.31004 \text {, }
$$

provided $\operatorname{dih} \geq \operatorname{dih}(S(2,2,2,2.51,2,2))$.

To deduce Lemma 9.5, we consider the piecewise linear bound $\ell$ on $\Gamma$ obtained from these estimates. The linear pieces are $\ell_{1}(x) \leq 1 \mathrm{pt}, \ell_{2}, \ell_{3}$, and $\ell_{4}$ on $\left[\mathrm{dih}_{\min }, d_{1}\right]$, $\left[d_{1}, d_{2}\right],\left[d_{2}, d_{3}\right]$, and $\left[d_{3}, \operatorname{dih}_{\max }\right]$, where $d_{1}=\operatorname{dih}(S(2,2,2,2,2,2)), d_{2}=1.42068$, and $d_{3}=\operatorname{dih}(S(2,2,2,2.51,2,2))$. (See Calculation 9.1 and Lemma 8.3.2.) Diagram 9.5.4 illustrates these linear bounds. (There are small discontinuities at $d_{1}, d_{2}$, and $d_{3}$ that may be eliminated by replacing $\ell_{i}$ by $\ell_{i}+\varepsilon_{i}$, for some $\varepsilon_{i}>0$, for $i=1,2$, and 4.) We then ask for the maximum of $\ell\left(t_{1}\right)+\ell\left(t_{2}\right)+\ell\left(t_{3}\right)+\ell\left(t_{4}\right)$ under the constraint $\left(t_{1}+t_{2}+t_{3}+t_{4}\right) / 4 \geq \pi / 2 \in\left[d_{2}, d_{3}\right]$. Since $\ell(x)$ is constant on $\left(\mathrm{dih}_{\min }, d_{1}\right)$ and decreasing on $\left[d_{1}, \operatorname{dih}_{\max }\right]$, we may assume that $t_{i} \geq d_{1}$, for all $i$. Since the slope of $\ell_{2}$ is equal to the slope of $\ell_{4}$, we may assume that $t_{i} \geq d_{2}$, for all $i$, or that $t_{i} \leq d_{3}$, for all $i$. If $t_{i} \leq d_{3}$, for all $i$, then we find that $t_{i} \geq 2 \pi-3 d_{3}>d_{2}$. So in either case, $t_{i} \geq d_{2}$, for all $i$. By convexity, an upper bound is $4 \ell_{3}(\pi / 2)<0.33 \mathrm{pt}$.

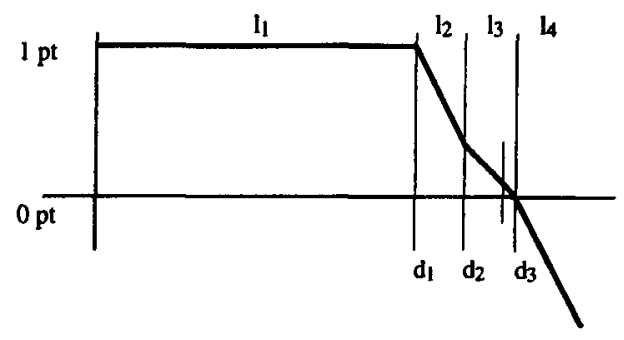

Diagram 9.5.4

Lemma 9.6. If $S_{1}, \ldots, S_{5}$ are any five tetrahedra such that $\operatorname{dih}\left(S_{1}\right)+\cdots+\operatorname{dih}\left(S_{5}\right) \geq$ $2 \pi$, then

$$
\Gamma\left(S_{1}\right)+\cdots+\Gamma\left(S_{5}\right)<4.52 p t
$$

This is a consequence of two other calculations. 
Calculation 9.6.1. $\Gamma<-0.207045 \mathrm{dih}+0.31023815$, provided dih $\in\left[d_{0}, 2 \pi-4 d_{0}\right]$, where $d_{0}=\operatorname{dih}(S(2,2,2,2,2,2))$.

Calculation 9.6.2. $\Gamma<0.028792018$, if dih $>2 \pi-4 d_{0}$.

Calculations 9.1, 9.6.1, and 9.6.2 give a piecewise linear bound $\ell$ on $\Gamma$ as a function of dihedral angle. See Diagram 9.6.3. (Again, there are minute discontinuities that may be eliminated in the same manner as before.) We claim that $4.52 p t>\ell\left(t_{1}\right)+\cdots+\ell\left(t_{5}\right)$ whenever $t_{1}+\cdots+t_{5} \geq 2 \pi$. As in Lemma 9.5 , we may assume that $t_{i} \geq d_{0}$. Since $\ell$ is decreasing on $\left[d_{0}, \operatorname{dih}_{\max }\right]$, we may assume that $t_{1}+\cdots+t_{5}=2 \pi$. Thus, only the interval $\left[d_{0}, 2 \pi-4 d_{0}\right]$ is relevant for the optimization. On this interval, the bound is linear, so $\ell\left(t_{1}\right)+\cdots+\ell\left(t_{5}\right) \leq 5 \ell(2 \pi / 5)<4.52 p t$.

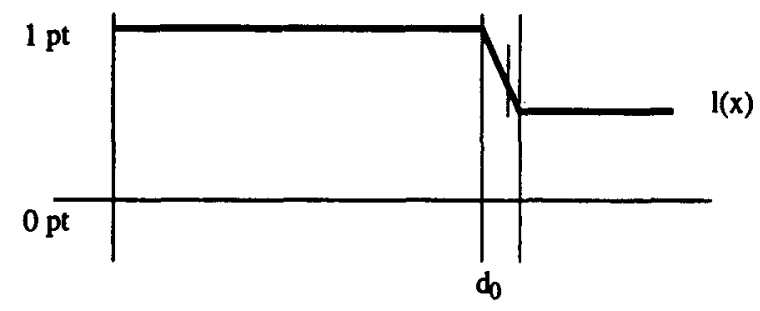

Diagram 9.6.3

Calculation 9.7. $\Gamma<0.389195 \operatorname{dih}-0.435643$, if $y_{1} \geq 2.05$.

The next inequalities relate the solid angles to the compression.

Calculation 9.8. $\quad \Gamma<-0.37642101$ sol +0.287389 .

Calculation 9.9. $\Gamma<0.446634 \mathrm{sol}-0.190249$.

Calculation 9.10. $\Gamma<-0.419351 \mathrm{sol}+0.2856354+0.001$, if $y_{i} \in[2,2.1]$, for $i=$ $1, \ldots, 6$.

The following calculation involves the circumradius. We leave it to the reader to check that the dimension-reduction techniques of Lemma 8.7.1 may still be applied.

Calculation 9.11. $\Gamma<-0.419351$ sol +0.2856354 , provided that $y_{4}, y_{5}, y_{6} \geq 2.1$ and that the circumradius of $S$ is at most 1.41.

Calculation 9.12. $\Gamma<-0.419351$ sol $+0.2856354-5(0.001)$, if $y_{i}>2.1$ for some $i$, and $y_{4} \in[2,21]$.

Calculation 9.13. $\Gamma<-0.65557 \mathrm{sol}+0.418$, if $y_{i} \in[2,2.1]$, for $i=1, \ldots, 6$. 
Calculation 9.14. $\operatorname{sol}(S)>0.21$.

Calculation 9.15. $\Gamma+(K-$ sol $) / 3<0.564978$ dih -0.614725 , where $K=$ $(4 \pi-6.48) / 12$, provided $y_{1} \in[2,2.05]$, and $y_{2}, y_{3} \in[2,2.2]$.

Calculation 9.16. dih $>0.98$ and sol $>0.45$, provided $y_{1} \in[2,2.05]$, and $y_{2}, y_{3} \in$ $[2,2.2]$.

Let $\Gamma(S)$ be replaced by $\operatorname{vor}(S)$ in each of the Calculations 9.1-9.16 to obtain a new list of inequalities $9.1^{\prime}-9.16^{\prime}$. (In $9.11^{\prime}$ we drop the constraint on the circumradius of $S$.) We claim that all of the inequalities 9.*' hold whenever $S$ is a quasi-regular tetrahedron of circumradius at least 1.41. In fact, inequalities $9.1^{\prime}, 9.2^{\prime}, 9.4^{\prime}, 9.5 .1^{\prime}, 9.5 .2^{\prime}, 9.5 .3^{\prime}, 9.6 .1^{\prime}$, 9.6.2' and $9.7^{\prime}$ follow directly from 9.17 and the inequalities $\operatorname{dih}_{\min } \leq \mathrm{dih} \leq \operatorname{dih}_{\max }$. Calculations 9.3, 9.14, and 9.16 are independent of $\Gamma$, and so do not require modification. Inequalities $9.8^{\prime}, 9.11^{\prime}$, and $9.12^{\prime}$ also rely on $9.17,9.18$, and 9.19 , inequality $9.9^{\prime}$ on 9.14 , and inequality $9.15^{\prime}$ on 9.16 . Inequalities $9.10^{\prime}$ and $9.13^{\prime}$ are vacuous by the comments of paragraph 8.2.4.

Write $S=S\left(y_{1}, y_{2}, y_{3}, y_{4}, y_{5}, y_{6}\right)$, vor $=\operatorname{vor}(S)$, and let $\operatorname{rad}=\operatorname{rad}(S)$ be the circumradius of $S$.

Lemma 9.17. If the circumradius is at least 1.41 , then vor $<-1.8 p t$.

Proof. If sol $\geq 0.91882$, then the lemma is a consequence of Lemma 9.18. (The proof of Lemma 9.18, under the restriction sol $\geq 0.91882$, is independent of the proof of this lemma.) Assume that sol $<0.91882$.

If $S^{\prime}$ and $S$ and Delaunay stars, related as in Section 8.7, then $\operatorname{vor}\left(S^{\prime}\right)>\operatorname{vor}(S)$ because $\hat{S}_{0}^{\prime}$ is obtained by slicing a slab from $\hat{S}_{0}$. This means that we may apply the dimension-reduction techniques described at the beginning of this section, unless the deformation decreases the circumradius to 1.41. An interval calculation establishes the result when $y_{1}+y_{2}+y_{3} \geq 6.3$ and the circumradius constraint is met (Calculation 9.17.1). When the dimension-reduction techniques apply, the dimension of the search space may be reduced to four, and an interval calculation similar to the others in this section gives the result (Calculation 9.17.2).

We lacked the computer resources to perform the interval analysis directly when the dimension-reduction techniques fail and found it necessary to break the problem up into smaller pieces when $y_{1}+y+2+y_{3} \leq 6.3$. We make use of the following calculations.

Calculation 9.17.1. $\operatorname{vor}(S)<-1.8 p t$ provided $y_{1}+y_{2}+y_{3} \geq 6.3, \operatorname{rad}(S)=1.41$, and $\operatorname{sol}(S) \leq 0.91882$.

Calculation 9.17.2. $\operatorname{vor}(S)<-1.8 p t$ provided that $\operatorname{rad}(S)>1.41, y_{1}+y_{2}+y_{3} \geq 6.3$, $\operatorname{sol}(S) \leq 0.912882$, and $S$ lies in one of the seven subspaces of smaller dimension associated with $I(\mathrm{sol}, \Gamma)$ at the beginning of the section. 
Calculation 9.17.3.1. Assume that $y_{1}+y_{2}+y_{3} \leq 6.3$ and that $\mathrm{rad} \geq 1.41$. Then sol $\geq 0.767$.

Calculation 9.17.3.2. Assume that $y_{1}+y_{2}+y_{3} \leq 6.192$ and that $\mathrm{rad} \geq 1.41$. Then sol $\geq 0.83$.

Calculation 9.17.3.3. Assume that $y_{1}+y_{2}+y_{3} \leq 6.106$ and that $\mathrm{rad} \geq 1.41$. Then sol $\geq 0.87$.

Calculation 9.17.3.4. Assume that $y_{1}+y_{2}+y_{3} \leq 6.064$ and that $\mathrm{rad} \geq 1.41$. Then sol $\geq 0.9$.

Calculation 9.17.3.5. Assume that $y_{1}+y_{2}+y_{3} \leq 6.032$ and that $\mathrm{rad} \geq 1.41$. Then sol $\geq 0.91882$.

In the interval arithmetic verification of Calculations 9.17.3, we may assume that $\mathrm{rad}=1.41$ and that $y_{1}+y_{2}+y_{3}$ is equal to the given upper bound 6.3, 6.192, etc. To see this we note that the circumradius constraint is preserved by a deformation of $S$ that increases $y_{1}, y_{2}$, or $y_{3}$ while keeping fixed the spherical triangle on the unit sphere at the origin cut out by $S$. We increase $y_{1}, y_{2}$, and $y_{3}$ in this way until the sum equals the given upper bound. Then fixing $y_{1}, y_{2}$, and $y_{3}$, and one of $y_{4}, y_{5}$, and $y_{6}$, we decrease the other two edges in such a way as to decrease the solid angle and circumradius until $\mathrm{rad}=1.41$.

This deformation argument would break down if we encountered a configuration in which two of $y_{4}, y_{5}$, and $y_{6}$ equal 2 , but this cannot happen when $\operatorname{rad}(S) \geq 1.41$ because this constraint on the edges would lead to the contradiction

$$
1.41 \leq \operatorname{rad}(S) \leq \operatorname{rad}(S(2.3,2.3,2.3,2,2,2.51))<1.39
$$

It is possible to reduce Calculations 9.17.3 further to the four-dimensional situation where two of $y_{4}, y_{5}$ and $y_{6}$ are either 2 or 2.51 . Consider a simplex $S$ with vertices $0, v_{1}$, $v_{2}$, and $v_{3}$. Let $p_{i}$ be the corresponding vertices of the spherical triangle cut out by $S$ on the unit sphere at the origin. Fix the origin, $v_{2}$, and $v_{3}$, and vary the vertex $v_{1}$. The locus on the unit sphere described as $p_{1}$ traces out spherical triangles of fixed area is an arc of a Lexell circle $\mathcal{C}$. Define the "interior" of $\mathcal{C}$ to be the points on the side of $\mathcal{C}$ corresponding to spherical triangles of smaller area. The locus traced by $v_{1}$ on the circumsphere (of $S$ ) with $\left|v_{1}\right|$ constant is a circle. Let $\mathcal{C}^{\prime}$, also a circle, be the radial projection of this locus to the unit sphere. Define the "interior" of $\mathcal{C}^{\prime}$ to be the points coming from larger $\left|v_{1}\right|$. The two circles $\mathcal{C}$ and $\mathcal{C}^{\prime}$ meet at $p_{1}$, either tangentially or transversely. The interior of $\mathcal{C}$ cannot be contained in the interior of $\mathcal{C}^{\prime}$ because the Lexell arc contains $p_{2}^{*}$, the point on the unit sphere antipodal to $p_{2}$ [FT, p. 23], but the interior of of $\mathcal{C}^{\prime}$ does not. Furthermore, if $\left|v_{1}\right|=2$ and $\left|v_{2}\right|+\left|v_{3}\right|>4$, then $v_{2}$ or $v_{3}$ lies in the interior of $\mathcal{C}$ and $\mathcal{C}^{\prime}$, so that the circles have interior points in common. This means that $v_{1}$ can always be moved in such a way that the solid angle is decreasing, the circumradius is constant, and the length $\left|v_{1}\right|$ is decreasing (or constant if $\left|v_{1}\right|=2$ ). If any two of $y_{4}, y_{5}$, and $y_{6}$ are not at an 
extreme point, this argument can be applied to $v_{1}, v_{2}$, or $v_{3}$ to decrease the solid angle. This proves the reduction.

We are now in a position to prove the lemma for simplices satisfying $y_{1}+y_{2}+y_{3} \leq 6.3$. Calculation 9.17.3.1 allows us to assume sol $\geq 0.767$. As in Section 8.6, let $S_{y}=$ $S(2,2,2, y, y, y)$. We rely on the fact that $\operatorname{vor}\left(S_{y}\right)$ is decreasing in $y$, for $y \in[2.26,2.41]$. In fact, the results of Section 8.6 specialize to the formula

$$
\operatorname{vor}\left(S_{y}\right)=\frac{-8 \delta_{o c t} y^{2}}{\left(12-y^{2}\right)^{1 / 2}\left(16-y^{2}\right)}+\frac{8}{3} \arctan \left(\frac{\left(12-y^{2}\right)^{1 / 2} y^{2}}{64-6 y^{2}}\right)
$$

and the sign of its derivative is determined by a routine Mathematica calculation. It is clear that $\operatorname{sol}\left(S_{y}\right)$ is continuous and increasing in $y$. Since $\operatorname{sol}\left(S_{2.26}\right)<0.767$ and $\operatorname{sol}\left(S_{2.41}\right)>$ 0.91882 , our conditions imply that $\operatorname{sol}(S)=\operatorname{sol}\left(S_{y}\right)$ for some $y \in[2.26,2.41]$. Let $r(a)$ and $\operatorname{vol}(R(a, b, c))$ be the functions introduced in Section 8.6.4.

This suggests the following procedure. Pick $y$ so that $\operatorname{sol}(S) \geq \operatorname{sol}\left(S_{y}\right)$. Calculate the smallest (or at least a reasonably small) $a$ for which

$$
\xi(y, a):=\operatorname{vor}\left(S_{y}\right)-4 \delta_{o c t}\left(1-\frac{1}{a^{3}}\right) 2 r(a)
$$

is less than -1.8 pt. Monotonicity (Calculation 9.20.1) and Lemma 8.6.5 imply that $\operatorname{vor}(S)<-1.8 p t$, if $y_{1}+y_{2}+y_{3} \geq 2(2+a)$. To treat the case that remains $\left(y_{1}+y_{2}+y_{3}<\right.$ $2(2+a))$, use Calculations 9.17 .3 to obtain a new lower bound on sol $(S)$, and hence a new value for $y$. The procedure is repeated until $a=0.016$. Calculation 9.17.3.5 completes the argument by covering the case $y_{1}+y_{2}+y_{3} \leq 6.032$. We leave it to the reader to check that

$$
\xi(2.2626,1.096), \xi(2.326,1.053), \xi(2.364,1.032), \xi(2.391,1.016)
$$

are less than $-1.8 p t$ and that

$$
\operatorname{sol}\left(S_{2.2626}\right) \leq 0.767, \operatorname{sol}\left(S_{2.326}\right) \leq 0.83, \operatorname{sol}\left(S_{2.364}\right) \leq 0.87, \operatorname{sol}\left(S_{2.391}\right) \leq 0.9 .
$$

This completes the proof of Lemma 9.17.

Lemma 9.18. If $\operatorname{rad}(S) \geq 1.41$, then vor $<-0.419351 \mathrm{sol}+0.2856354$.

Proof. We adopt the notation and techniques of Lemma 9.17. If sol $\leq 0.918819$, then the result follows from Lemma 9.17. (The proof of Lemma 9.17 is independent of the argument that follows under that restriction on solid angles.) Let $f(S)=$ $-0.419351 \mathrm{sol}(S)+0.2856354-\operatorname{vor}(S)$. We show that $f(S)$ is positive. We use the inequality $f(S) \geq f\left(S_{\text {tan }}\right)+4 \delta_{\text {oct }} \operatorname{vol}\left(\hat{S} \backslash \hat{S}_{\text {tan }}\right)$ of Lemma 8.6.4. A routine calculation based on formula (9.17.4) shows that $f\left(S_{y}\right)$ is increasing, for $y \in[2.4085,2.51]$.

If sol $\geq 0.951385$, then we appeal to Fejes Tóth's convexity argument described in Section 8.6. To justify the use of his argument, we must verify that the cone over $S_{\text {tan }}$ contains the circumcenter of $S$. The first, second, and third edges have length 2 , and the fourth, fifth, and sixth edges are between 2.21 and 2.51 (Calculation 9.18.2). These are 
stronger restrictions on the edges than in the proof of Lemma 8.6.5, so the justification there applies here as well. We observe that $\operatorname{sol}\left(S_{2.4366}\right)<0.951385$ so that

$$
f(S) \geq f\left(S_{\text {tan }}\right) \geq f\left(S_{y}\right) \geq f\left(S_{2.4366}\right) \approx 0.000024>0,
$$

where $y$ satisfies $\operatorname{sol}\left(S_{y}\right)=\operatorname{sol}\left(S_{\mathrm{tan}}\right)$. We may assume that $0.918819 \leq \operatorname{sol} \leq 0.951385$.

Lemma 9.18.1. The combined volume of the two Rogers simplices along a common edge of a quasi-regular tetrahedron $S$ is at least 0.01 .

Proof. The combined volume is at least that of the right-circular cone of height $a$ and base a wedge of radius $\sqrt{b^{2}-a^{2}}$ and dihedral angle $\operatorname{dih}_{\min }$, where $a$ is the half-length of an edge and $b$ is a lower bound on the circumradius of a face with an edge $2 a$. This gives the lower bound of

$$
\frac{\pi}{3}\left(b^{2}-a^{2}\right) a \frac{\mathrm{dih}_{\min }}{2 \pi}>0.1439\left(b^{2}-a^{2}\right) .
$$

We minimize $b$ by setting $b^{2}=\eta(2,2,2 a)^{2}=4 /\left(4-a^{2}\right)$. Then $b^{2}-a^{2}=$ $\left(a^{2}-2\right)^{2} /\left(4-a^{2}\right)$, which is decreasing in $a \in[1,2.51 / 2]$. Thus, we obtain a lower bound on $b^{2}-a^{2}$ by setting $a=2.51 / 2$, and this gives the estimate of the lemma.

We remark that $\operatorname{sol}\left(S_{2.4085}\right)<0.918819$. If $1.15 \leq\left(y_{1}+y_{2}+y_{3}-4\right) / 2$, then Lemma 9.18.1 and Section 8.6 give

$$
f(S) \geq f\left(S_{2.4085}\right)+4 \delta_{\text {oct }}\left(1-\frac{1}{1.15^{3}}\right) 0.01>0 .
$$

(Analytic continuation is not required here, because of the constraints on the edges in Calculation 9.18.2.) We may now assume that $y_{1}+y_{2}+y_{3} \leq 6.3$. To continue, we need a few more calculations.

Calculation 9.18.2. If sol $\geq 0.918$, then $y_{4}, y_{5}, y_{6} \geq 2.21$.

Calculation 9.18.3.1. If $y_{1}+y_{2}+y_{3} \leq 6.02$ and rad $\geq 1.41$, then sol $\geq 0.928$.

Calculation 9.18.3.2. If $y_{1}+y_{2}+y_{3} \leq 6.0084$ and $\mathrm{rad} \geq 1.41$, then $\mathrm{sol} \geq 0.933$.

Calculation 9.18.3.3. If $y_{1}+y_{2}+y_{3} \leq 6.00644$ and $\mathrm{rad} \geq 1.41$, then sol $\geq 0.942$.

In the verification of these calculations, we make the same reductions as in Calculations 9.17.3.

Adapting the procedure of Lemmas 9.17 and 8.6.5, we fine that a lower bound on the solid angle leads to an estimate of a constant $a$ with the property that $f(S)>0$ whenever $y_{1}+y_{2}+y_{3} \geq 4+2 a$. That is, we pick $a$ so that $\xi_{1}(y, a):=f\left(S_{y}\right)+8 \delta_{o c t}\left(1-1 / a^{2}\right) r(a)$ 
is positive, where $y$ is chosen so that $\operatorname{sol}\left(S_{y}\right)$ is a lower bound on the solid angle. The values

$$
\xi_{1}(2.4085,1.01), \xi_{1}(2.4165,1.0042), \xi_{1}(2.42086,1.00322), \xi_{1}(2.4286,1.0017)
$$

are all positive. This yields the bound $y_{1}+y_{2}+y_{3} \leq 6.0034$.

Assume that $S$ satisfies $y_{1}+y_{2}+y_{3} \leq 6.0034$ and $\operatorname{rad}(S) \geq 1.41$. Then by Calculation 9.18.3.3, $\operatorname{sol}(S)=\operatorname{sol}\left(S_{\mathrm{tan}}\right) \geq 0.942$. Also $\operatorname{rad}\left(S_{\mathrm{tan}}\right)>\operatorname{rad}(S) / 1.0017 \geq$ $1.41 / 1.0017>1.4076$, because rescaling $S_{\tan }$ by a factor of 1.0017 gives a simplex containing $S$. This means that $S_{\tan }$ satisfies the hypotheses of the following calculation. Calculation 9.18.4 completes the proof of Lemma 9.18.

Calculation 9.18.4. If $\operatorname{rad}\left(S_{\tan } \geq 1.4076\right.$ and $0.933 \leq \operatorname{sol}\left(S_{\tan }\right) \leq 0.951385$, then $f\left(S_{\text {tan }}\right)>0$.

In this verification we may assume that the fourth, fifth, and sixth edges of $S_{\text {tan }}$ are at least 2.27 , for otherwise the circumradius is at most

$$
\operatorname{rad}(S(2,2,2,2.27,2.51,2.51))<1.4076
$$

In the verification of Calculation 9.18.4, we also rely on the fact that

$$
f_{1}\left(x_{4}, x_{5}, x_{6}\right):=f\left(S\left(2,2,2, \sqrt{x_{4}}, \sqrt{x_{5}}, \sqrt{x_{6}}\right)\right)
$$

is increasing in $\left(x_{4}, x_{5}, x_{6}\right) \in\left[2.27^{2}, 2.51^{2}\right]$. Here is a sketch justifying this fact. The details were carried out in Mathematica with high-precision arithmetic. The explicit formulas of Section 8.6 lead to an expression for $\partial f_{1} / \partial x_{4}$ as

$$
\frac{W\left(x_{4}, x_{5}, x_{6}\right)\left(-x_{4}+x_{5}+x_{6}\right)}{\left(-16+x_{4}\right)^{2} \Delta^{3 / 2}}
$$

where $W$ is a polynomial in $x_{4}, x_{5}$, and $x_{6}$ (with 13 terms). To show that $W$ is positive, expand it in a Taylor polynomial about $\left(x_{4}, x_{5}, x_{6}\right)=\left(2.27^{2}, 2.27^{2}, 2.27^{2}\right)$ and check that the lower bound of inequality $(7.1)$ is positive.

Calculation 9.19. If $y_{4} \in[2,2.1]$, then sol $<0.906$.

For $a \leq b \leq c$, we have $\operatorname{vol}(R(a, b, c))=a\left(\left(b^{2}-a^{2}\right)\left(c^{2}-b^{2}\right)\right)^{1 / 2} / 6$. The final four calculations are particularly simple (to the extent that any of these calculations are simple), since they involve a single variable. They were verified in Mathematica with rational arithmetic.

Calculation 9.20.1. The function $\left(1-1 / a^{2}\right) \operatorname{vol}(R(a, \eta(2,2,2 a), 1.41))$ is increasing on $[1,1.15]$.

Calculation 9.20.2. The function $\operatorname{vol}(R(a, \eta(2,2,2 a), 1.41))$ is decreasing for $a \in$ $[1,1.15]$. 
Calculation 9.20.3. For $a \in[1,1.15]$ we have

$$
\operatorname{vol}(R(a, \eta(2,2,2 a), 1.41))<\operatorname{vol}(R(a, \eta(2,2.51,2 a), 1.41)) .
$$

Calculation 9.21. Let $p_{1}=p_{1}\left(y_{1}, \ldots, y_{6}\right)$ be the point in Euclidean space introduced in Section 8.6.7. For $y \in[2.3,2.51]$,

$$
\frac{d}{d y}\left(\eta(2.51,2.51, y)-\frac{y^{2}}{4}\right)^{1 / 2}>-0.75>\frac{d}{d y}\left(\left|p_{1}(y, 2.51,2,2,2,2.51)\right|^{2}-\frac{y^{2}}{4}\right)^{1 / 2}
$$

\section{Acknowledgments}

I would like to thank D. J. Muder for the Appendix and the referees for suggesting other substantial improvements.

\section{Appendix. Proof of Theorem 6.1}

\section{J. Muder}

Notation and Observations. Let $P$ be a point of degree $d$. If we consider $P$ to be the center of the configuration, then the first rim of points will be the set of $d$ points adjacent to $P$, and the second rim will be those at distance 2 from $P$. Let $\delta(P)$ be the sum of the degrees of the first rim points. If $d=6$, it is easy to see that the number of points on the second rim is $s=\delta(P)-24$, and the total number of points of distance at most 2 from $P$ is $\delta(P)-17$. The second rim is thus an $s$-gon. This $s$-gon and the triangulation of the $P$-side of the $s$-gon are referred to as the inner graph. The inner-graph degree of a second rim point is called its inner degree. The number of second-rim points with inner degree 5 is equal to the number of degree 4 points in the first rim, and the number of second-rim points with inner degree 3 is equal to the number of degree 6 points on the first rim. All other second-rim points have inner degree 4. Points beyond the second rim are called extra points. If there are no extra points, then there are at least two second-rim points whose degrees are equal to their inner degrees. Let $N_{\Delta}^{i}(P)$ be the number of points of degree $\Delta$ in rim $i$ around $P$. Notice that if $d=6$, then

$$
\delta(P)=30-N_{4}^{1}(P)+N_{6}^{1}(P) .
$$

Further, Euler's formula gives $N-12=N_{6}-N_{4}$. Putting this together with 5.1.1 and 5.1.5, we see

$$
\begin{gathered}
13 \leq 12+N_{6}-N_{4} \leq 15 \\
1 \leq 1+N_{4} \leq N_{6} \leq 3+N_{4} \leq 5 .
\end{gathered}
$$

Lemma 1. There is no triangle whose vertices all have degree 6. 
Lemma 2. Suppose $N_{6} \geq 3$. Let $\mathbf{G}_{6}$ be the subgraph of points of degree 6 . Either $\mathbf{G}_{6}$ is three points in two components, or it is a single (open or closed) path, with no other edges.

With these lemmas and this notation, we consider the different possible values of $N_{6}$.

$N_{6}=1$. This forces $N_{4}=0$. Let $P$ be the degree 6 point. Then $\delta(P)=30$, all thirteen points are in the inner graph, and all six second-rim points have inner degree 4 . However, at least two of these second-rim points have inner degree equal to degree. Contradiction.

$N_{6}=2$. If the two degree 6 points are adjacent, let $P$ be either one. Now $\delta(P)=31-N_{4}^{1}$, and we see that there are no extra points, and all second-rim points have degree 5 . All but $N_{4}^{1}$ second-rim points have inner degree less than 5 , and at least two of them will have inner degree equal to degree. So $N_{4} \geq N_{4}^{1}=2$, and $N \leq 12$. Contradiction.

If the degree 6 points are not adjacent, then the $(6,6)$ forces $N \geq 14$, so $N_{4}=0$. If $\delta(P)$ is either of the two degree 6 points, $\delta(P)=30$, so the second rim has six points, all of inner degree 4 , and there is one extra point. If the extra point has degree 5 , it is adjacent to all but one of the second-rim points. This unique second-rim point is then part of a quadrilateral, which can only be triangulated by the diagonal edge that does not include the extra point. This creates two second-rim points of degree 6 , in violation of our assumptions. Therefore the extra point has degree 6 , and we have Diagram 6.2.

$N_{6}=3$. Either $\mathbf{G}_{6}$ is an open path or it has two components. In either case there is a $(6,6)$, so $N \geq 14$ and $N_{4}=0$ or 1 .

In the first case let $P$ be the center of the path. Now $\delta(P)=32-N_{4}^{1}$. We see that there are no extra points and any degree 4 point is in the first rim. The second rim has at most one point of inner degree greater than 4 , and two points whose inner degrees are equal to their degrees. So there must be a point of degree 4 on the second rim. Contradiction.

In the second case 5.1.8 forces $N=15$, hence $N_{4}=0$. If $P$ is one of the points in the two-point component of $\mathbf{G}_{6}$, then $\delta(P)=31$, so there is one extra point and seven second-rim points. Either one or two of the second rim points is not connected to the extra point. In either case at least one of these two has degree = inner degree, which is at most 4 . Contradiction.

$N_{6}=4$. Now $\mathbf{G}_{6}$ is either an open or closed path of length 4. If open, then, by 5.1.8, $N=15$ and $N_{4}=1$. If $P$ is either of the interior degree 6 points, then $\delta(P)=32-N_{4}^{1}$, the second rim is an $\left(8-N_{4}^{1}\right)$-gon with $N_{4}^{1}$ points of inner degree at least 5 and $N_{4}^{1}$ extra points. We cannot have $N_{4}^{1}=0$, or else there would be at least two second-rim points of degree 4 , so $N_{4}^{1}=1$. This holds no matter which interior degree 6 point we started with, so the degree 4 point must be adjacent to both. It must also be adjacent to one of the other two, or else there is a $(6,6,4)$. Therefore there is a degree 6 point whose firstrim degree sequence is $6 / 4 / 6 / 5 / 5 / 5$, producing a second-rim inner degree sequence of $4 / 3 / 5 / 3 / 4 / 4 / 4$. The point of inner degree 5 is the fourth neighbor of the degree 4 point, and cannot be degree 6 without making $\mathbf{G}_{6}$ closed. Therefore there is an edge connecting the two points of inner degree 3 . Adding this edge to the inner graph creates a hexagon with all points of "inner" degree 4 , and the extra point in its interior. The extra point is connected to five of these points, and the sixth has degree 4. Contradiction. 
If $\mathbf{G}_{6}$ is a closed path, then a degree 4 point $Q$ must triangulate this quadrilateral. Using $Q$ as the center, there are four first-rim points and eight second-rim points. The second rim can be drawn in a square with the four points of inner degree 3 on the corners and the four points of inner degree 4 at the midpoints. There is at most one additional degree 4 point (other than $Q$ ). If it is anywhere but at one of the second-rim midpoints, a $(6,6,4)$ exists. The four midpoints cannot be adjacent to any additional second-rim points without creating an illegal triangle or quadrilateral. Since there can be at most one more degree 4 point, at least three of the four second-rim midpoints must be joined to the extra point(s). Two midpoints of consecutive sides cannot be joined to the same extra point without forcing the corresponding corner to be degree 4 , which is impossible. So there must be two extra points, and thus no degree 4 points other than $Q$. One extra point must be joined to the midpoints of each pair of parallel sides of the square. However, all these edges cannot be drawn without intersecting.

$N_{6}=5$. Now $\mathbf{G}_{6}$ is a path of length 5 , closed so that a $(6,6,6)$ does not exist. Any point in this pentagon is nonadjacent to two others, so the situation of 5.1.8 applies in five different ways. Let $P, Q, R \in \mathbf{G}_{6}$ with $P$ and $Q$ adjacent, but neither adjacent to $R$. Then there are eight points adjacent to either $P$ or $Q$, and six points adjacent to $R$, so there are exactly two common points in these two sets. In our case these two points are precisely the other two points of degree 6 . This means that no point inside or outside the pentagon can be connected to more than two points of the pentagon. Thus the only possible configuration for the 15 points is to form three concentric pentagons, with $\mathbf{G}_{6}$ as the middle one. Prior to triangulating the inner and outer pentagons, all of their points have degree 4 . Triangulating either creates more degree 6 points. Contradiction.

Proof of Lemma 1. Let $P_{1}, P_{2}$, and $P_{3}$ be the vertices of such a triangle. If $P_{j}$ has no neighbors of degree 4 , then the only possibility is $\delta\left(P_{j}\right)=32$, which forces $N_{5}^{1}=4$, $N_{6}^{1}=2$, and $N=15$. All second-rim points have inner degree at most 4 , and there are no extra points. Therefore two second-rim points have degree and inner degree 4 . Call them $Q_{1}$ and $Q_{2}$. Therefore, $N_{4} \geq 2, N_{6} \geq 5$, and $N_{6}^{2} \geq 2$. Let $R_{1}$ and $R_{2}$ be second-rim points of degree 6. Each $Q_{k}$ and $R_{\ell}$ must be adjacent, or else $\left(P_{j}, R_{\ell}, Q_{k}\right)$ is a $(6,6,4)$. However, all edges from $Q_{j}$ are inner-graph edges. So $Q_{1} R_{1} Q_{2} R_{2}$ must be a second-rim quadrilateral, which is impossible. Thus $N_{4}^{1}\left(P_{j}\right) \geq 1$ for each $P_{j}$.

This forces $N_{4}=2$. At least one of the degree 4 points is adjacent to two of the $P_{j}$. One of these two, say $P_{1}$, is not adjacent to the other degree 4 point. Since $\delta(P) \leq$ 32 , we are left with four possible first-rim degree sequences for $P_{1}$ : (1) $6 / 6 / 4 / 5 / 5 / 5$; (2) $6 / 6 / 4 / 6 / 5 / 5$; (3) $6 / 6 / 4 / 5 / 6 / 5$ : or (4) $6 / 6 / 4 / 5 / 5 / 6$.

The last three possibilities are easily dealt with. Sequence (3) contains a $(6,6,4)$. Sequence (4) creates two 6-6-6 triangles joined at an edge. Let $P_{1}$ and $P_{2}$ be the vertices of the edge, and let $P_{3}$ and $P_{4}$ be the other vertices of the triangles. The conditions $N_{4}^{l}\left(P_{j}\right) \geq 1$ and $N_{4}=2$ force $P_{3}$ and $P_{4}$ to have sequence (1). In (2) we can invoke 5.1.8 with the three degree 6 points in the first rim. Call them $R_{1}, R_{2}$, and $T$, with $R_{1}$ adjacent to $R_{2}$. In order for the numbers to work out, there can be precisely two points which are adjacent to $T$ as well as one of the $R_{j}$. However, in (2) the center, the first-rim degree 4 point, and the second-rim neighbor of the first-rim degree 4 point must all fit this description. 
Sequence (1) is more difficult to eliminate. Let $R_{1}, \ldots, R_{6}$ be the first-rim points listed in the order of (1). Let $S_{1} \ldots S_{7}$ be the second-rim points, with $S_{1}$ being adjacent to both $R_{1}$ and $R_{2}$, and $S_{7}$ having only $R_{1}$ as a first-rim neighbor. There is at least one degree 6 point in the second rim-if it is at $S_{1}$, then $\delta\left(R_{1}\right)=32$; if it is anywhere else we can invoke 5.1.8. In either case, $N=15$ and there are two degree 6 points outside the first rim. These must lie in $\left\{S_{6}, S_{7}, S_{1}, S_{3}\right\}$ to avoid making a $(6,6,4)$ with $R_{1}$ and $R_{3}$. At most one of these points can be adjacent to $R_{1}$ (since $\delta\left(R_{1}\right)<33$ ), so $S_{3}$ must have degree 6 . Now $S_{3}$ must connect to both the degree 6 and degree 4 points in $\left\{S_{6}, S_{7}, S_{1}\right\}$ to avoid making a $(6,6,6)$ or $(6,6,4)$ with the center point. However, $S_{3}$ has inner degree 5 and so can connect to at most one of those points. Contradiction.

Proof of Lemma 2. If $\mathbf{G}_{6}$ has at least three points and no triangles, then there exists a $(6,6)$ forcing $N \geq 14$. Any point in $\mathbf{G}_{6}$ can be adjacent to at most two others, or else either a triangle or a $(6,6,6)$ is created. Therefore each component is a path. There cannot be three or more components without producing a $(6,6,6)$. If $\mathbf{G}_{6}$ has two components, each of them must be a complete graph for the same reason. Since there are no triangles, both of the components must have at most two points. If there are two components with two points each, then 5.1.8 forces $N=15$. Each component is adjacent to eight non- $\mathbf{G}_{6}$ points of the original graph, and not adjacent to three. Now 5.1 .8 forces each point in the other component to be adjacent to these three. However, two points can have only two common neighbors. So the only two-component $\mathbf{G}_{6}$ is the one described in the lemma.

\section{References}

[AH] G. Alefeld and J. Herzeberger, Introduction to Interval Computations, Academic Press, New York, 1983.

[FT] L. Fejes Tóth, Lagerungen in der Ebene, auf der Kugel und im Raum, Springer-Verlag, Berlin, 1953.

[H1] T. C. Hales, Remarks on the density of sphere packings in three dimensions, Combinatorica, 13(2) (1993), 181-197.

[H2] T. C. Hales, The sphere packing problem, J. Comput. Appl. Math, 44 (1992), 41-76.

[H3] T. C. Hales, The status of the Kepler conjecture, Math. Intelligencer, 16(3) (1994), 47-58.

[H4] T. C. Hales, Sphere packings, II, Discrete Comput. Geom., to appear.

[HS] T. C. Hales, Sphere packings, $\Pi \mathrm{L}_{\alpha}$, in preparation.

[H6] T. C. Hales, Packings, http://www-personal.math.lsa.umich.edu/ hales/packings.html

[H7] J. F. Hart et al., Computer Approximations, Wiley, New York, 1968.

[IEEE] IEEE Standard for Binary Floating-Point Arithmetic, ANSI/IEEE Std. 754-1985, IEEE, New York.

[P] W. H. Press et al., Numerical recipes in C, Less-Numerical Algorithms, second edition, Cambridge University Press, Cambridge, 1992. Chapter 20.

[R] C. A. Rogers, The packing of equal spheres, Proc. London Math. Soc. (3) 8 (1958), 609-620.

[W] What every computer scientist should know about floating-point arithmetic, Comput. Surveys, 23(1) (1991), 5-48. 